\title{
Link Homologies and the Refined Topological Vertex
}

\author{
Sergei Gukov ${ }^{1}$, Amer Iqbal ${ }^{2}$, Can Kozçaz ${ }^{3}$, Cumrun Vafa ${ }^{4,5}$ \\ 1 Department of Physics, University of California, Santa Barbara, CA, 93106, USA \\ 2 Department of Physics, LUMS School of Science \& Engineering, \\ U Block, D.H.A, Lahore, Pakistan. E-mail: amer.iqbal@lums.edu.pk \\ 3 Department of Physics, University of Washington, Seattle, WA, 98195, USA \\ 4 Center for Theoretical Physics, Massachusetts Institute of Technology, \\ Cambridge, MA, 02139, USA \\ 5 Jefferson Physical Laboratory, Harvard University, Cambridge, MA, 02138, USA
}

Received: 16 September 2009 / Accepted: 14 December 2009

Published online: 20 April 2010 - (C) The Author(s) 2010. This article is published with open access at Springerlink.com

\begin{abstract}
We establish a direct map between refined topological vertex and $\operatorname{sl}(N)$ homological invariants of the of Hopf link, which include Khovanov-Rozansky homology as a special case. This relation provides an exact answer for homological invariants of the Hopf link, whose components are colored by arbitrary representations of $s l(N)$. At present, the mathematical formulation of such homological invariants is available only for the fundamental representation (the Khovanov-Rozansky theory) and the relation with the refined topological vertex should be useful for categorizing quantum group invariants associated with other representations $\left(R_{1}, R_{2}\right)$. Our result is a first direct verification of a series of conjectures which identifies link homologies with the Hilbert space of BPS states in the presence of branes, where the physical interpretation of gradings is in terms of charges of the branes ending on Lagrangian branes.
\end{abstract}

\section{Contents}

1. Introduction . . . . . . . . . . . . . . . . 758

2. BPS States, Link Invariants, and Open Topological Strings . . . . . . . . . . 760

2.1 Geometric transition and the Hopf link . . . . . . . . . . . . . . . . . 762

2.2 Knots, links and open topological string amplitudes . . . . . . . . . . . 762

3. Link Homologies and Topological Strings . . . . . . . . . . . . . . . . . 765

3.1 Hopf link: the fundamental representation . . . . . . . . . . . . . . . 768

4. Refined Topological Vertex . . . . . . . . . . . . . . . . . 768

4.1 Open topological string amplitudes . . . . . . . . . . . . . . 771

4.1.1 Hopf link. . . . . . . . . . . . . . . . . . . . . . . . 771

5. Refined Vertex and Link Homologies . . . . . . . . . . . . . . . . . . . . . . . . . . . . . . . . . . .

5.1 Unknot. . . . . . . . . . . . . . . . . . 775

5.2 Hopf link . . . . . . . . . . . . . . . . . . . 776

A. Appendix: Other Representations . . . . . . . . . . . . . . . . . 778 
A.1 Unknot. . . . . . . . . . . . . . . . . . 778

A.2 Hopf link . . . . . . . . . . . . . . . . . . 780

A.3 Specialization to $Q=-t q^{-2 N}$ : Some examples . . . . . . . . . 782

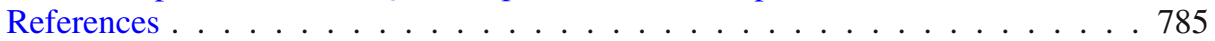

\section{Introduction}

One of the most promising recent developments in a deeper understanding of link invariants involves the study of homological invariants. First, these invariants provide a refinement of the familiar polynomial invariants. Secondly, and more importantly, they often lift to functors. However, constructing such homological invariants for arbitrary groups and representations has been a challenging problem, and at present only a handful of link homologies is known. Most of the existing examples are related to the fundamental representation of classical groups of type $A$ and include the Khovanov homology [1], the link Floer homology [2-4], and the $s l(N)$ knot homology $[5,6]$.

On the physics side, polynomial invariants of knots and links can be realized in the Chern-Simons gauge theory [7]. On the other hand, a physical interpretation of link homologies was first proposed in [8] and further developed in $[9,10]$. The interpretation involves BPS states in the context of physical interpretation of open topological string amplitudes [11]. In order to explain the realization in topological string theory one first needs to consider embedding the Chern-Simons gauge theory in string theory [12] and the large $N$ dual description in terms of topological strings [13]. As was shown in [11] and will be reviewed in the next section, in this dual description polynomial invariants of knots and links are mapped to open topological string amplitudes which, in turn, can be reformulated in terms of integer enumerative invariants counting degeneracy of states in Hilbert spaces, roughly the number of holomorphic branes ending on Lagrangian branes. This leads to a physical reformulation of polynomial link invariants in terms of the so-called Ooguri-Vafa invariants which, roughly speaking, compute the Euler characteristic of the $Q$-cohomology, that is cohomology with respect to the nilpotent components of the supercharge. ${ }^{1}$

This, however, is not the full answer to homological link invariants which require the understanding of an extra grading. In other words, there is an extra physical charge needed to characterize these invariants. In closed string theory, an extension of topological string was constructed for certain non-compact Calabi-Yau geometries [14]. It involves an extra parameter which has the interpretation of an extra rotation in the fourdimensional space. It was shown in [15] that this extra charge indeed accounts for the charges of the M2 branes on holomorphic curves inside a Calabi-Yau three-fold.

It was proposed in [8] that the homological grading of link homologies is related to the extra charge in the extension of topological string proposed in [14]. In particular, supersymmetric states of holomorphic branes ending on Lagrangian branes, labeled by all physical charges, should reproduce homological invariants of knots and links,

$$
\mathcal{H}(L)=\mathcal{H}_{B P S}
$$

This conjecture led to a number of predictions regarding the structure of $s l(N)$ knot homologies, in particular to the triply-graded knot homology categorifying the HOMFLY polynomial $[9,16]$, see also [10]. However, a direct test of this conjecture and

\footnotetext{
1 Elements of this cohomology can be viewed as the ground states of the supersymmetric theory of M2 branes ending on M5 branes in a particular geometry [11], as we review below.
} 
Table 1. Enumerative invariants of Calabi-Yau three-folds

\begin{tabular}{llll}
\hline & Rational & Integer & Refinement \\
\hline $\begin{array}{l}\text { Closed } \\
\text { Open }\end{array}$ & Gromov-Witten & Gopakumar-Vafa/Donaldson-Thomas & $\begin{array}{c}\text { Refined BPS invariants } \\
\text { Open Gromov-Witten }\end{array}$ \\
& Ooguri-Vafa invariants & $D_{J, s, r}$ and $N_{J, s, r}$ \\
\hline
\end{tabular}

computation of homological link invariants from string theory was difficult due to lack of techniques suitable for calculating degeneracies of BPS states in the physical setup.

Thus, even for the unknot, the only case where one can compute both sides of (1) independently is the case of the fundamental representation. For other representations, a mathematical formulation of homological knot invariants is not available at present, while on the string theory side the direct analysis of $\mathcal{H}_{B P S}$ becomes more difficult. For a certain class of representations - which, for example, include totally symmetric and totally anti-symmetric representations of $s l(N)$ - it was argued in [10] that the corresponding cohomology ring of the unknot, $\mathcal{H}^{\mathbf{g}, R}$, is related to the Jacobi ring of a potential $W_{\mathbf{g}, R}\left(x_{i}\right)$,

$$
\mathcal{H}^{\mathbf{g}, R}(\text { unknot }) \cong \mathcal{J}\left(W_{\mathbf{g}, R}\left(x_{i}\right)\right) .
$$

It is expected that for this class of representations the corresponding link homologies can be defined using matrix factorizations of the potential $W_{\mathbf{g}, R}\left(x_{i}\right)$, as in the original construction of the Khovanov and Rozansky [6]. The simplest set of examples of such representations involves totally anti-symmetric representations of $s l(N)$. For the the $k^{\text {th }}$ antisymmetric representation of $s l(N)$, the potential is the Landau-Ginzburg potential of the $A_{N}^{\otimes k}$ minimal model, and the corresponding homology ring of the unknot (2) is the cohomology ring of the Grassmannian of $k$-planes in $\mathbb{C}^{N}[6,10]$,

$$
\mathcal{H}^{s l(N), \Lambda^{k}}(\text { unknot }) \cong H^{*}(\operatorname{Gr}(k, N)),
$$

where all cohomology groups are localized in the single homological grading. This will be one of our examples below.

We will be able to compute the homology groups $\mathcal{H}^{\mathrm{g}, R}$ directly from string theory using the recent work [17], where it was shown how the topological vertex [18] (which computes topological string amplitudes in toric geometries (Table 1)) can be refined to compute Refined BPS invariants [15]. Since the topological vertex formalism is composed of open string amplitudes, this refinement together with the conjecture of [8] implies that the refined topological vertex should be computing homological link invariants, at least for the class of links which can be formulated in terms of local toric geometries. The basic example of such a link is the Hopf link. This is one of the few examples where we can directly verify our conjectures, at least in the case of the fundamental representation, where Khovanov-Rozansky homology of the Hopf link can be computed. We find in this paper that these highly non-trivial computations agree with each other exactly!

This provides a strong check of the various conjectures leading to this statement. Moreover, since the refined topological vertex is easily computable for arbitrary representations, this leads to a prediction of all homological invariants of a large class of links (of which the Hopf link is the simplest example) colored by arbitrary representations $\left(R_{1}, \ldots, R_{\ell}\right)$,

$$
\mathcal{H}^{s l(N) ; R_{1}, \ldots, R_{\ell}}(L)
$$


This is a highly non-trivial new prediction which we are currently studying, and it would be very interesting to compare it with the mathematical formulation of link homologies, once those are developed. It is likely that these predictions lead to a deeper mathematical understanding of homological link invariants. In particular, we hope that the combinatorial interpretation of the refined vertex in terms of 3D partitions will be useful for finding the combinatorial definition of link homologies (4).

The organization of this paper is as follows: In Sect. 2 we review the relation between the BPS state counting, link invariants, and open topological strings, including the large $N$ description of the Chern-Simons theory. In Sect. 3 we review aspects of homological link invariants and their interpretation as Hilbert spaces of BPS states. In particular, we use this interpretation to compute the Khovanov-Rozansky homology of the Hopf link. In Sect. 4 we review the refined topological vertex, which is used in Sect. 5 - together with some facts from Sect. 2 - to compute the homological invariants for the Hopf link colored by arbitrary representations $\left(R_{1}, R_{2}\right)$, see Eq. (67) below. In particular, in the case of the fundamental representation we reproduce the Khovanov-Rozhansky homology derived in Sect. 3, and make new predictions.

Conventions. The triply-graded invariants discussed in this paper are naturally organized into generating functions, which are polynomials in three variables. Unfortunately, the conventions between the physics literature and the knot theory literature are slightly different. In order to be careful about such differences and to agree with the standard notations, we use the variables $\left(Q, q_{1}, q_{2}\right)$ when we talk about topological string amplitudes computed by the topological vertex, $c f$. [17], and we use the variables $(\mathbf{a}, q, t)$ when we discuss link homologies, $c f$. [9]. The two sets of variables are related as follows:

$$
\begin{aligned}
\sqrt{q_{2}} & =q, \\
\sqrt{q_{1}} & =-t q, \\
Q & =-t \mathbf{a}^{-2} .
\end{aligned}
$$

In particular, expressions written in terms of $(\mathbf{a}, q, t)$ involve integer powers of $q$ and $t$, while expressions written in terms of $\left(Q, q_{1}, q_{2}\right)$ involve half-integer powers of $q_{1}$ and $q_{2}$. Specialization to the Ooguri-Vafa invariants and to knot polynomials is achieved, respectively, by setting $q_{1}=q_{2}$ and $t=-1$.

\section{BPS States, Link Invariants, and Open Topological Strings}

For the benefit of the reader not very familiar with the description of D-branes in toric varieties, following [19,20], let us briefly review the basics of this description necessary for understanding the topological string interpretation of link homologies. Consider a toric variety,

$$
X=\mathbf{C}^{k+3} / U(1)^{k}
$$

where $\mathbf{C}^{k+3}$ is parametrized by coordinates $X^{i}, i=1, \ldots, k+3$, and the symplectic quotient is obtained by imposing

$$
\begin{aligned}
& D^{a}=Q_{1}^{a}\left|X^{1}\right|^{2}+Q_{2}^{a}\left|X^{2}\right|^{2}+\cdots+Q_{k+3}^{a}\left|X^{k+3}\right|^{2}-r^{a}=0, \\
& U(1)_{a}: \quad X^{i} \rightarrow e^{i Q_{i}^{a} \epsilon_{a}} X^{i}
\end{aligned}
$$




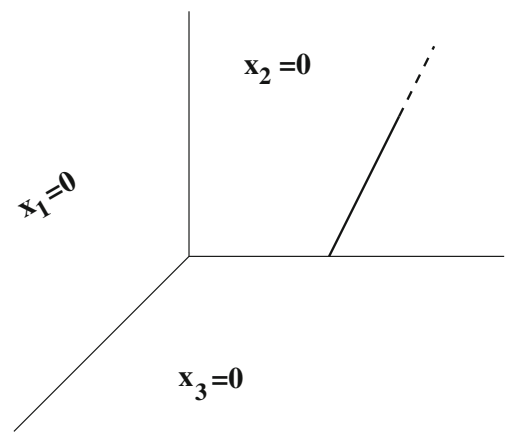

Fig. 1. A Lagrangian D-brane in $\mathbf{C}^{3}$, projected to the base of the toric fibration

for every $a=1, \ldots, k$. We can think of (6) as a gauged linear sigma model with gauge group $U(1)^{k}$ and chiral fields $X^{i}$ of charges $Q_{i}^{a}$. The charges $Q_{i}^{a}$ should obey

$$
\sum_{i} Q_{i}^{a}=0
$$

Using toric geometry, we can also describe Lagrangian D-branes invariant under the torus action. There are two interesting types of Lagrangian D-branes:

1. Lagrangians, which project to a 1-dimensional subspace in the base of the toric variety $X$. These can be described by three equations of the form:

$$
\begin{aligned}
\sum_{i} q_{i}^{\alpha}\left|X_{i}\right|^{2} & =c^{\alpha}, \quad \alpha=1,2, \\
\sum_{i} \arg X_{i} & =0,
\end{aligned}
$$

where $q_{i}^{\alpha}$ is a set of charges such that $\sum_{i} q_{i}^{\alpha}=0$.

2. Lagrangians, which project to a 2-dimensional subspace in the base of the toric variety $X$. These can be defined by the following equations:

$$
\begin{aligned}
\sum_{i} q_{i}^{1}\left|X_{i}\right|^{2} & =c, \\
\sum_{i} q_{i}^{\alpha} \arg X_{i} & =0, \quad \alpha=2,3,
\end{aligned}
$$

where the charges should satisfy $\sum_{i} q_{i}^{1} q_{i}^{\alpha}=0, \alpha=2,3$.

Let us consider $X=\mathbf{C}^{3}$ with a Lagrangian D-brane on $L$, where $L$ is defined by

$$
\begin{aligned}
& \left|X_{1}\right|^{2}-\left|X_{3}\right|^{2}=c>0, \\
& \left|X_{2}\right|^{2}-\left|X_{3}\right|^{2}=0, \\
& \sum_{i} \arg X_{i}=0 .
\end{aligned}
$$

The projection of this Lagrangian D-brane to the base of toric fibration is shown on Fig. 1. 
2.1. Geometric transition and the Hopf link. The conjecture on the geometric transition [13] was originally checked at the level of free energies and later at the level of observables of the theory in more detail in [11]. A worldsheet explanation of this duality was discovered in [21]. See [22] for a detailed review of this duality and its consequences for link invariants.

Let us briefly review the conjectured equivalence between the Chern-Simons theory in $\mathbf{S}^{3}$ with the closed topological string theory on the resolved conifold, or in other words, with the open topological string theory on $T^{*} \mathbf{S}^{3}$.

In his work, 't Hooft noted that $U(N)$ or $S U(N)$ gauge theories should have a string theory description. If we consider the perturbative Feynman diagram expansion in the 't Hooft coupling $\lambda=N g$ using the double line notation, these diagrams can be regarded as a triangulation of a Riemann surface. The contributions to the free energy coming from these diagrams can be arranged in a way that looks like open string expansion on worldsheet with genus $g$ and $h$ boundaries:

$$
F=\sum_{g=0, h=1} C_{g, h} N^{2-2 g} \lambda^{2 g-2+h} .
$$

It was shown by Witten for the $S U(N)$ Chern-Simons theory on a three dimensional manifold $\mathbf{S}^{3}$ that the coefficients $C_{g, h}$ are equal to the A-model topological open string theory on a worldsheet with genus $g$ and $h$ boundaries [12] with the target space $T^{*} \mathbf{S}^{3}$. The $N$ D-branes are wrapped on the base $\mathbf{S}^{3}$ in this six dimensional cotangent bundle. The summation over the number of holes in Eq. 11 can be carried out first. The free energy takes the following form which looks like the closed string expansion:

$$
F=\sum_{g=0} N^{2-2 g} F_{g}(\lambda),
$$

where $\lambda$ acts like some modulus of the theory. The natural question that arises is "what is the closed string theory for the Chern-Simons theory on $\mathbf{S}^{3}$ ?" In [13] it was conjectured that if we start with the open topological string theory on $T^{*} \mathbf{S}^{3}$ which can be regarded as the deformed conifold and wrap $N$ D-branes on the base and take the large $N$ limit, the geometry of the target space undergoes the conifold transition: the base $\mathbf{S}^{3}$ shrinks and then is blown up to $\mathbf{S}^{2}$, where the D-branes disappear. Instead, the Kähler moduli of the blown up $\mathbf{S}^{2}$ is proportional to the 't Hooft coupling. The equivalence was checked for all values of the 't Hooft coupling and for all genera of the free energy of the Chern-Simons theory and the closed topological strings on the resolved conifold.

It is worth mentioning that the resolution of the geometry, however, is not unique: two different ways of resolving the singularity give rise to topologically distinct spaces which are birationally equivalent. In Fig. 5, two different resolutions of the conifold singularity are shown which are related by flop. If we insert probe branes in the target geometry and compute the open string partition function using the "usual" topological vertex the partition function is invariant under flop. However, for the "refined" topological vertex this invariance does not hold, and it will be crucial in our discussion to choose the 'correct' blowup.

2.2. Knots, links and open topological string amplitudes. The equivalence between the open topological string on the deformed conifold and the closed string on the resolved conifold was also checked in terms of the observables [11]. The basic observables in the 
Chern-Simons theory are the Wilson loops. As mentioned before, there are $N$ D-branes wrapped on the base, and to study their dynamics another set of D-branes can be introduced, say $M$ of them. This new set of D-branes will be wrapped on a Lagrangian 3 -cycle which is associated with a knot. A closed loop $q(s),(0 \leq s<2 \pi)$, is used to parametrize a knot in $\mathbf{S}^{3}$. Then the conormal bundle associated with the knot defined as

$$
\mathcal{C}=\left\{(q(s), p) \mid p^{i} \frac{d q_{i}}{d s}=0,0 \leq s<2 \pi\right\}
$$

is Lagrangian. The $M$ D-branes wrapped on the Lagrangian cycle $\mathcal{C}$ gives rise to $S U(M)$ Chern-Simons theory. However, in addition to the Chern-Simons theory on $\mathcal{C}$ there is another topological open string sector coming from strings stretching between the $M$ D-branes around $\mathcal{C}$ and the $N$ D-branes around the base $\mathbf{S}^{3}$. We obtain a complex scalar which transforms as a bi-fundamental of $S U(N) \otimes S U(M)$ and lives in the intersection of the D-branes, i.e. on the knot. This complex field can be integrated out and we obtain an effective action for the $U(N)$ gauge connection $A$ on $\mathbf{S}^{3}$,

$$
S_{C S}(A)+\sum_{n=1}^{\infty} \frac{1}{n} \operatorname{Tr} U^{n} \operatorname{Tr} V^{-n},
$$

which can be rephrased as correlations of [23]

$$
\left\langle\sum_{R} \operatorname{Tr}_{R} U T r_{R} V^{-1}\right\rangle
$$

In the previous section we mentioned that the geometry changes from deformed conifold with branes to the resolved conifold without branes if we take the large $N$ limit. We can take the same limit in this brane system while keeping the number of non-compact probe branes, $M$, fixed and trace what happens to the probe branes during this transition. According to [11], the non-compact Lagrangian cycle $\mathcal{C}$ will be mapped to a new Lagrangian cycle $\mathcal{C}^{\prime}$ in the resolved conifold, with $M$ D-branes wrapping it. This will provide boundary conditions for the open strings to end on in the resolved geometry. Aspects of this transition including how one can find the Lagrangian brane for certain knots and links (including the Hopf link) have been discussed in [24]. Precise mathematical description of the Lagrangian D-brane $\mathcal{C}^{\prime}$ after transition has been offered [25].

For the case of the unknot, discussed in detail in [11], the normalized CS expectation is given by

$$
W_{\lambda(R)}=\left\langle\operatorname{Tr}_{R} U\right\rangle, \quad U=P e^{\oint A},
$$

where $\lambda(R)$ is the highest weight of the irreducible representation $R$, i.e., it is a $2 \mathrm{D}$ partition. The above expectation value can be calculated exactly and is given by

$$
\begin{aligned}
& W_{\lambda}=\text { Quantum dimension of } \lambda=\prod_{(i, j) \in \lambda} \frac{q_{1}^{\frac{N+c(i, j)}{2}}-q_{1}^{-\frac{N+c(i, j)}{2}}}{q_{1}^{\frac{h(i, j)}{2}}-q_{1}^{-\frac{h(i, j)}{2}}} \\
& =q_{1}^{-N \frac{|\lambda|}{2}} s_{\lambda}\left(1, q_{1}, q_{1}^{2}, \ldots, q_{1}^{N-1}\right) \text {, }
\end{aligned}
$$




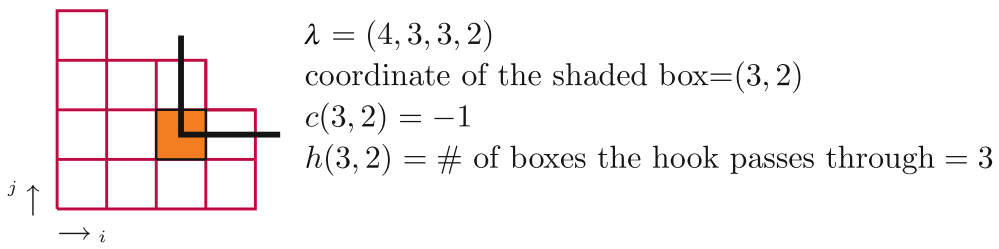

Fig. 2. The content and the hook length of a box in a Young diagram

where $s_{\lambda}(\mathbf{x})$ is the Schur function labelled by the partition $\lambda$ and $c(i, j)=j-i$, $h(i, j)=\lambda_{i}-j+\lambda_{j}^{t}-i+1$ are the content and the hook length of a box in the Young diagram of $\lambda$ as shown in Fig. 2 .

Similarly for the Hopf link we can color the two component knots by two different representations to obtain

$$
W_{\lambda \mu}=\left\langle\operatorname{Tr}_{\lambda} U_{1} \operatorname{Tr}_{\mu} U_{2}\right\rangle,
$$

where $U_{1}$ and $U_{2}$ are the two holonomy matrices around two component unknots. This can also be calculated exactly to obtain

$$
W_{\lambda \mu}=q_{1}^{\frac{\kappa(\mu)}{2}} s_{\lambda}\left(q_{1}^{-\rho}\right) s_{\mu}\left(q_{1}^{-\rho-\lambda}, Q q_{1}^{\rho}\right) \prod_{(i, j) \in \lambda}\left(1-Q q_{1}^{i-j}\right) .
$$

Here $Q=q_{1}^{-N}$. We will recall the geometry of D-branes for the unknot and Hopf link in Sect. 4 and review how the open topological string amplitudes in the presence of these branes reproduce the above knot and link invariants, before extending it to more refined invariants.

In [11] the open topological string amplitudes were interpreted as counting a certain BPS partition function. This interpretation is crucial for connecting it to link homologies as the Hilbert space is naturally in the problem. Moreover the gradation of the homology is nothing but the charges of BPS states in the physical theory. The geometry considered in [11] was as follows: We can lift the type IIA geometry of the resolved conifold to M-theory. In this context the probe branes get mapped to M5 branes wrapping the Lagrangian cycles and filling the non-compact $R^{3}$ spacetime. The open topological string simply computes the number of M2 branes ending on the M5 branes. The representation of the link invariant encodes the geometry of the ending of the M2 brane on the M5 brane. Moreover the coefficient of $q^{s} Q^{J}$ in the topological string amplitudes, $N_{R, J, s}$, is determined by the number of such bound states which wrap the $\mathbb{P}^{1} J$ times and have spin $s$ under the $\mathrm{SO}(2)$ rotation of the spatial $\mathbb{R}^{2} \subset \mathbb{R}^{3}$. $^{2}$ The precise structure of the connection between open topological strings and BPS counting was further elaborated in [27], to which we refer the interested reader. For a single knot, for example, one finds that the free energy $F=\log (Z)$ as a function of $V$ defined above, is given by

$$
F(V)=-\sum_{R, n>0} f_{R}\left(q^{n}, Q^{n}\right) \frac{\operatorname{Tr}_{R} V^{n}}{n},
$$

where $f_{R}(q, Q)$ is completely determined by the BPS degeneracies of the M2 brane, $N_{R^{\prime}, J, s}$, where $R^{\prime}$ denotes the representation the BPS state transforms in $J$, is the charge

\footnotetext{
${ }^{2}$ For a complete mathematical proof of the integrality of $N_{R, J, s}$ see [26].
} 
of the brane and $s$ is the spin. Moreover the sign of $N$ is correlated with its fermion number.

It was proposed in [8] that there is a further charge one can consider in labeling the BPS states of M2 branes ending on M5 branes: The normal geometry to the M5 brane includes, in addition to the spacetime $\mathbb{R}^{3}$, and the three normal directions inside the $\mathrm{CY}$, an extra $\mathbb{R}^{2}$ plane. It was proposed there that the extra $S O(2)$ rotation in this plane will provide an extra gradation which could be viewed as a refinement of topological strings and it was conjectured that this is related to link homologies that we will review in the next section. This gives a refinement of $N_{R, J, s} \rightarrow N_{R, J, r, s}$. In other words for a given representation $R$ we have a triply graded structure labeling the BPS states.

\section{Link Homologies and Topological Strings}

Now, let us proceed to describing the properties of link homologies suggested by their relation to Hilbert spaces of BPS states. We mostly follow notations of [8,9].

Let $L$ be an oriented link in $\mathbf{S}^{3}$ with $\ell$ components, $K_{1}, \ldots, K_{\ell}$. We shall consider homological as well as polynomial invariants of $L$ whose components are colored by representations $R_{1}, \ldots, R_{\ell}$ of the Lie algebra $\mathbf{g}$. Although in this paper we shall consider only $\mathbf{g}=\operatorname{sl}(N)$, there is a natural generalization to other classical Lie algebras of type $B, C$, and $D$. In particular, there are obvious analogs of the structural properties of $\operatorname{sl}(N)$ knot homologies for $s o(N)$ and $\operatorname{sp}(N)$ homologies (see [10,28] for some work in this direction).

Given a link colored by a collection of representations $R_{1}, \ldots, R_{\ell}$ of $s l(N)$, we denote the corresponding polynomial invariant by

$$
\bar{P}_{s l(N) ; R_{1}, \ldots, R_{\ell}}(q) \text {. }
$$

Here and below, the "bar" means that (20) is the unnormalized invariant; its normalized version $P_{S l(N) ; R_{1}, \ldots, R_{\ell}}(q)$ obtained by dividing by the invariant of the unknot is written without a bar. Since this "reduced" version depends on the choice of the "preferred" component of the link $L$, below we mainly consider a more natural, unnormalized invariant (20). In the special case when every $R_{a}, a=1, \ldots, \ell$ is the fundamental representation of $\operatorname{sl}(N)$ we simply write

$$
\bar{P}_{N}(q) \equiv \bar{P}_{s l(N) ; \square, \ldots, \square}(q) .
$$

The polynomial invariants (20) are related to expectation values of Wilson loop operators $W(L)=W_{R_{1}, \ldots, R_{\ell}}(L)$ in Chern-Simons theory. For example, the polynomial $\operatorname{sl}(N)$ invariant $P_{N}(q)$ is related to the expectation value of the Wilson loop operator $W(L)=W_{\square, \ldots, \square}(L)$,

$$
\bar{P}_{N}(L)=q^{-2 N 1 \mathrm{k}(L)}\langle W(L)\rangle,
$$

where $\operatorname{lk}(L)=\sum_{a<b} \operatorname{lk}\left(K_{a}, K_{b}\right)$ is the total linking number of $L$.

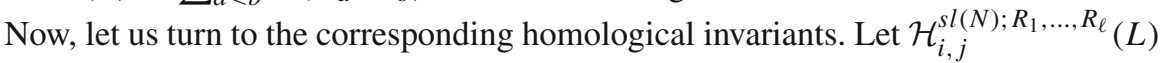
be the doubly-graded homology theory whose graded Euler characteristic is the polynomial invariant $\bar{P}_{s l(N) ; R_{1}, \ldots, R_{\ell}}(q)$,

$$
\bar{P}_{s l(N) ; R_{1}, \ldots, R_{\ell}}(q)=\sum_{i, j \in \mathbb{Z}}(-1)^{j} q^{i} \operatorname{dim} \mathcal{H}_{i, j}^{s l(N) ; R_{1}, \ldots, R_{\ell}}(L) .
$$


The graded Poincaré polynomial,

$$
\overline{\mathcal{P}}_{s l(N) ; R_{1}, \ldots, R_{\ell}}(q, t):=\sum_{i, j \in \mathbb{Z}} q^{i} t^{j} \operatorname{dim} \mathcal{H}_{i, j}^{s l(N) ; R_{1}, \ldots, R_{\ell}}(L)
$$

is, by definition, a polynomial in $q^{ \pm 1}$ and $t^{ \pm 1}$ with integer non-negative coefficients. Clearly, evaluating (24) at $t=-1$ gives (23).

When $R_{a}=\square$ for all $a=1, \ldots, \ell$, the homology $\mathcal{H}_{i, j}^{s l(N) ; R_{1}, \ldots, R_{\ell}}(L)$ is the Khovanov-Rozansky homology, $\overline{H K R}_{i, j}^{N}(L)$, and

$$
\begin{aligned}
\overline{K h R}_{N}(q, t) & \equiv \overline{\mathcal{P}}_{s l(N) ; \square, \ldots, \square}(q, t) \\
& =\sum_{i, j \in \mathbb{Z}} q^{i} t^{j} \operatorname{dim} \overline{H K R}_{i, j}^{N}(L)
\end{aligned}
$$

is its graded Poincaré polynomial.

The physical interpretation of homological link invariants via Hilbert spaces of BPS states leads to certain predictions regarding the behavior of link homologies with rank $N$. In particular, the total dimension of $\mathcal{H}_{*, *}^{s l(N) ; R_{1}, \ldots, R_{\ell}}(L)$ grows as

$$
\operatorname{dim} \mathcal{H}_{*, *}^{s l(N) ; R_{1}, \ldots, R_{\ell}}(L) \sim N^{d}, \quad N \rightarrow \infty,
$$

where

$$
d=\sum_{i=1}^{\ell} \operatorname{dim} R_{i}
$$

More specifically, a general form of the conjecture in [8] states:

Conjecture. There exists a "superpolynomial", $\overline{\mathcal{P}}_{R_{1}, \ldots, R_{\ell}}(\mathbf{a}, q, t)$, a rational function ${ }^{3}$ in three variables a, $q$, and $t$, such that

$$
\overline{\mathcal{P}}_{s l(N) ; R_{1}, \ldots, R_{\ell}}(q, t)=\overline{\mathcal{P}}_{R_{1}, \ldots, R_{\ell}}\left(\mathbf{a}=q^{N}, q, t\right)
$$

for sufficiently large $N$.

The coefficients of the superpolynomial, say, in the case of the fundamental representation:

$$
\overline{\mathcal{P}}_{N}(\mathbf{a}, q, t)=\frac{1}{\left(q-q^{-1}\right)^{\ell}} \sum_{J, s, r} \mathbf{a}^{J} q^{s} t^{r} D_{J, s, r}
$$

encode the dimensions of the Hilbert space of states, related to BPS states,

$$
D_{J, s, r}:=(-1)^{F} \operatorname{dim} \mathcal{H}_{B P S}^{F, J, s, r},
$$

graded by the fermion number $F$, the membrane charge $J$, and the $U(1)_{L} \times U(1)_{R}$ quantum numbers $s$ and $r$. However, note that the $D_{J, s, r}$ is not the same as $N_{J, s, r}: N_{J, s, r}$

\footnotetext{
3 This definition differs slightly from the ones introduced in [9], where it is the numerator of the rational function $\overline{\mathcal{P}}_{R_{1}, \ldots, R_{\ell}}(\mathbf{a}, q, t)$ which was called the superpolynomial. Since in general one has very good control of the denominators, the two definitions are clearly related.
} 
encodes the integral structure in the Free energy, whereas $D_{J, s, r}$ is the exponentiated version of it. It is not difficult to see that the integrality of $N_{J, s, r}$ guarantees that of $D_{J, s, r}$ (as in the closed string case where the integrality of GV invariants implies integrality of the DT invariants). This in particular explains that the Hilbert space structure of BPS states captured by $N_{J, s, r}$ will indeed encode the Hilbert space structure for $D_{J, s, r}$ and thus its integrality. However, it is not completely obvious from the physical picture why (28) is a finite polynomial, for any given $N$, as has been conjectured.

The conjecture (28) can be refined even further. Indeed, the large $N$ growth described in (26) and (28) is characterized by the contribution of individual link components,

$$
\oplus_{a=1}^{\ell} \mathcal{H}_{*, *}^{s l(N) ; R_{a}}\left(K_{a}\right)
$$

Often, it is convenient to remove this contribution and consider only the "connected" part of the polynomial (resp. homological) link invariant. For example, in the simplest case when all components of the link $L$ carry the fundamental representation, the corresponding $s l(N)$ invariant $\bar{P}_{N}(L)$ or, equivalently, the Wilson loop correlation function (22) can be written in terms of the integer BPS invariants $N_{(\square, \ldots, \square), Q, s}$ as

$$
\langle W(L)\rangle^{(c)}=\left(q^{-1}-q\right)^{\ell-2} \sum_{J, s} N_{(\square, \ldots, \square), J, s} q^{N J+s},
$$

where $\langle W(L)\rangle^{(c)}$ is the connected correlation function. Thus, for a two-component link, we have

$$
\langle W(L)\rangle^{(c)}=\langle W(L)\rangle-\left\langle W\left(K_{1}\right)\right\rangle\left\langle W\left(K_{2}\right)\right\rangle
$$

and

$$
\bar{P}_{N}(L)=q^{-2 N \operatorname{lk}(L)}\left[\bar{P}_{N}\left(K_{1}\right) \bar{P}_{N}\left(K_{2}\right)+\sum_{J, s} N_{(\square, \square), J, s} q^{N J+s}\right]
$$

where $\bar{P}_{N}\left(K_{1}\right)$ and $\bar{P}_{N}\left(K_{2}\right)$ denote the unnormalized $s l(N)$ polynomials of the individual link components.

Similarly, the homological $s l(N)$ invariant of a two-component link $L$ can be written as a sum of connected and disconnected terms [8]:

$$
\begin{aligned}
\overline{K h R}_{N}(L)= & q^{-2 N \operatorname{lk}(L)}\left[t^{\alpha} \overline{K h R}_{N}\left(K_{1}\right) \overline{K h R}_{N}\left(K_{2}\right)\right. \\
& \left.+\frac{1}{q-q^{-1}} \sum_{J, s, r \in \mathbb{Z}} D_{J, s, r} q^{N J+s} t^{r}\right],
\end{aligned}
$$

where integer invariants $D_{J, s, r}(L)$ are related to the dimensions of the Hilbert space of BPS states, $N_{J, s, r}$ and $\alpha$ is a simple invariant of $L$. At $t=-1$ this expression specializes to (34). 
3.1. Hopf link: the fundamental representation. The Hopf link, $L=2_{1}^{2}$ consists of two components, $K_{1} \cong K_{2} \cong$ unknot, which are linked with the linking number $\operatorname{lk}\left(K_{1}, K_{2}\right)=-1$. The $\operatorname{sl}(2)$ homological invariant for the Hopf link is

$$
\overline{K h R}_{2}\left(2_{1}^{2}\right)=1+q^{2}+q^{4} t^{2}+q^{6} t^{2} .
$$

It can be written in the form (35) with the following non-zero invariants:

$$
\begin{gathered}
D_{0,-1,0}=1, \quad D_{0,1,2}=-1, \\
D_{-2,-1,0}=-1, \quad D_{-2,1,2}=1 .
\end{gathered}
$$

This gives the "superpolynomial" for the Hopf link,

$$
\overline{\mathcal{P}}\left(2_{1}^{2}\right)=\frac{1}{\left(q-q^{-1}\right)^{2}}\left[\left(q^{-2}-1+q^{2} t^{2}\right)+\mathbf{a}^{2}\left(1-q^{2} t^{2}-q^{-2}-t^{2}\right)+\mathbf{a}^{4} t^{2}\right],
$$

which after specializing to $\mathbf{a}=q^{N}$ gives the graded Poincare polynomial of the $\operatorname{sl}(N)$ link homology:

$$
\begin{aligned}
\overline{K h R}_{N}\left(2_{1}^{2}\right)= & q^{N-1}\left(\frac{q^{N}-q^{-N}}{q-q^{-1}}\right)+q^{2 N}\left(\frac{q^{N}-q^{-N}}{q-q^{-1}}\right)^{2} t^{2} \\
& -q^{N+1}\left(\frac{q^{N}-q^{-N}}{q-q^{-1}}\right) t^{2} .
\end{aligned}
$$

Notice that at $t=-1$ this expression reduces to the correct formula for the $\operatorname{sl}(N)$ polynomial invariant of the Hopf link,

$$
\bar{P}_{N}\left(2_{1}^{2}\right)=1-q^{2 N}+q^{2 N}\left(\frac{q^{N}-q^{-N}}{q-q^{-1}}\right)^{2} .
$$

The result (39) agrees with the direct computation of Khovanov-Rozansky homology for small values of $N$ :

$$
\begin{aligned}
\overline{K h R}_{3}\left(2_{1}^{2}\right)= & 1+q^{2}+q^{4}+q^{4} t^{2}+2 q^{6} t^{2}+2 q^{8} t^{2}+q^{10} t^{2} \\
\overline{K h R}_{4}\left(2_{1}^{2}\right)= & 1+q^{2}+q^{4}+q^{4} t^{2}+q^{6}+2 q^{6} t^{2}+3 q^{8} t^{2}+3 q^{10} t^{2}+2 q^{12} t^{2}+q^{14} t^{2}, \\
\overline{K h R}_{5}\left(2_{1}^{2}\right)= & 1+q^{2}+q^{4}+q^{4} t^{2}+q^{6}+2 q^{6} t^{2}+q^{8}+3 q^{8} t^{2}+4 q^{10} t^{2}+4 q^{12} t^{2} \\
& +3 q^{14} t^{2}+2 q^{16} t^{2}+q^{18} t^{2} .
\end{aligned}
$$

\section{Refined Topological Vertex}

In this section we will briefly explain the combinatorial interpretation of the refined vertex in terms of 3D partitions; more details can be found in [17].

Recall that the generating function of the 3D partitions is given by the MacMahon function,

$$
\begin{aligned}
M(q) & =\sum_{n \geq 0} C_{n} q^{n}=\prod_{k=1}^{\infty}\left(1-q^{n}\right)^{-n}, \\
C_{n} & =\# \text { of } 3 \mathrm{D} \text { partitions with } n \text { boxes. }
\end{aligned}
$$




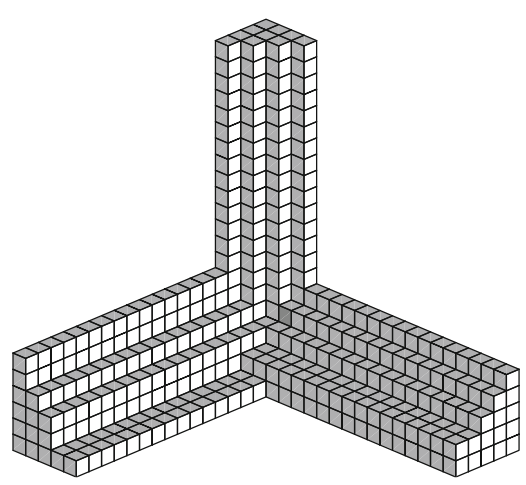

(a)

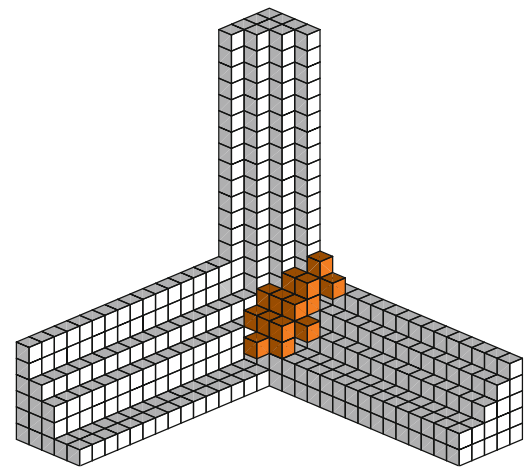

(b)

Fig. 3. (a) $\pi_{\bullet}(\lambda, \mu, v)$ for $\lambda=(6,4,3,1,1), \mu=(5,4,3,2,2), v=(4,3,2,1)$. (b) An example of $\pi(\lambda, \mu, v)$

The topological vertex $C_{\lambda \mu \nu}(q)[18]$,

$$
C_{\lambda \mu \nu}(q)=q^{\frac{\kappa(\mu)}{2}} s_{v^{t}}\left(q^{-\rho}\right) \sum_{\eta} s_{\lambda^{t} / \eta}\left(q^{-\rho-v}\right) s_{\mu / \eta}\left(q^{-\rho-v^{t}}\right),
$$

has the following combinatorial interpretation [30]:

$$
M(q) C_{\lambda \mu \nu}(q)=f_{\lambda \mu \nu}(q) \sum_{\pi(\lambda, \mu, \nu)} q^{|\pi(\lambda, \mu, \nu)|-\left|\pi_{\bullet}(\lambda, \mu, \nu)\right|}
$$

where $\pi(\lambda, \mu, v)$ is a $3 \mathrm{D}$ partition such that along the three axis which asymptotically approaches the three 2D partitions $\lambda, \mu$ and $\nu .|\pi|$ is number of boxes (volume) of the 3D partition $\pi$ and $\pi_{\bullet}$ is the $3 \mathrm{D}$ partition with the least number of boxes satisfying the same boundary condition. ${ }^{4}$ Figure 3 (a) shows the $\pi_{\bullet}$ for $\lambda=(6,4,3,1,1), \mu=(5,4,3,2,2)$ and $v=(4,3,2,1)$. Figure 3(b) shows an example of the partition $\pi(\lambda, \mu, v)$ for $\lambda, \mu, v$ the same as in Fig. 3(a). $f_{\lambda \mu \nu}(q)$ is the framing factor which appears because of the change from perpendicular slicing of the $3 \mathrm{D}$ partition to diagonal slicing of the $3 \mathrm{D}$ partition [30].

The refined topological vertex [17]

$$
\begin{aligned}
C_{\lambda \mu \nu}\left(q_{1}, q_{2}\right)= & \left(\frac{q_{1}}{q_{2}}\right)^{\frac{\|\mu\|^{2}-|\mu|}{2}} q_{2}^{\frac{\kappa(\mu)}{2}} q_{2}^{\frac{\|v\|^{2}}{2}} \widetilde{Z}_{v}\left(q_{1}, q_{2}\right) \\
& \times \sum_{\eta}\left(\frac{q_{2}}{q_{1}}\right)^{\frac{|\eta|+|\lambda|-|\mu|}{2}} s_{\lambda^{t} / \eta}\left(q_{1}^{-\rho} q_{2}^{-v}\right) s_{\mu / \eta}\left(q_{2}^{-\rho} q_{1}^{-v^{t}}\right)
\end{aligned}
$$

also has a similar combinatorial interpretation in terms of 3D partitions which we will explain now. Recall that the diagonal slices of a 3D partition, $\pi$, are 2D partitions which interlace with each other. These are the 2D partitions living on the planes $x-y=a$

\footnotetext{
4 Since even the partition with the least number of boxes, has infinite number of boxes, we need to regularize this by putting it in an $N \times N \times N$ box as discussed in [30].
} 


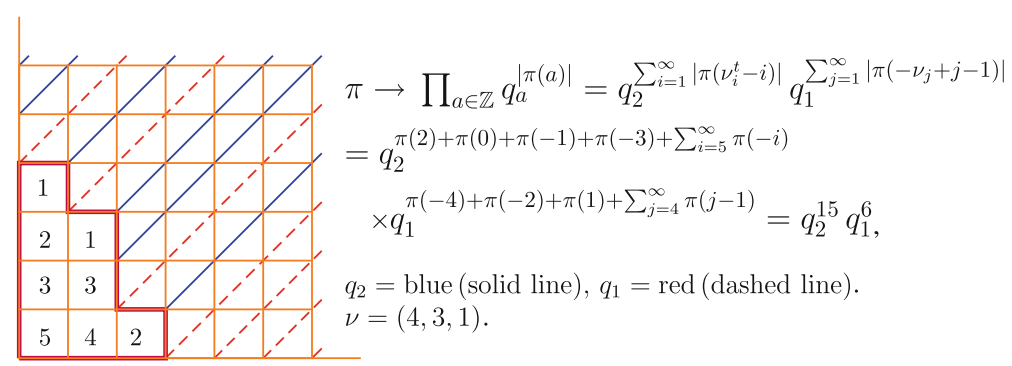

Fig. 4. Slices of the $3 D$ partitions are counted with parameters $q_{1}$ and $q_{2}$ depending on the shape of $v$

where $a \in \mathbb{Z}$. We will denote these $2 \mathrm{D}$ partitions by $\pi_{a}$. For the usual vertex the $\mathrm{a}^{\text {th }}$ slice is weighted with $q^{\left|\pi_{a}\right|}$, where $\left|\pi_{a}\right|$ is the number of boxes cut by the slice (the number of boxes in the $2 \mathrm{D}$ partition $\pi_{a}$ ). The $3 \mathrm{D}$ partition is then weighted by

$$
\prod_{a \in \mathbb{Z}} q^{\left|\pi_{a}\right|}=q^{\sum_{a \in \mathbb{Z}}\left|\pi_{a}\right|}=q^{\# \text { of boxes in the } \pi} .
$$

In the case of the refined vertex the $3 \mathrm{D}$ partition is weighted in a different manner. Given a 3D partition $\pi$ and its diagonal slices $\pi_{a}$ we weigh the slices for $a<0$ with parameter $q$ and the slices with $a \geq 0$ with parameter $t$ so that the measure associated with $\pi$ is given by

$$
\left(\prod_{a<0} q_{2}^{\left|\pi_{a}\right|}\right)\left(\prod_{a \geq 0} q_{1}^{\left|\pi_{a}\right|}\right)=q_{2}^{\sum_{i=1}^{\infty}|\pi(-i)|} q_{1}^{\sum_{j=1}^{\infty}|\pi(j-1)|}
$$

The generating function for this counting is a generalization of the MacMahon function and is given by

$$
M\left(q_{1}, q_{2}\right):=\sum_{\pi} q_{2}^{\sum_{i=1}^{\infty}|\pi(-i)|} q_{1}^{\sum_{j=1}^{\infty}|\pi(j-1)|}=\prod_{i, j=1}^{\infty}\left(1-q_{1}^{j} q_{2}^{i-1}\right)^{-1} .
$$

We can think of this assignment of $q_{1}$ and $q_{2}$ to the slices in the following way. If we start from large positive $a$ and move toward the slice passing through the origin, then every time we move the slice towards the left we count it with $q_{1}$ and every time we move the slice up (which happens when we go from $a=i$ to $a=i-1, i=0,1,2 \ldots$ ) we count it with $q_{2}$.

Since we are slicing the skew 3D partitions with planes $x-y=a$ we naturally have a preferred direction given by the $\mathrm{z}$-axis. We take the $2 \mathrm{D}$-partition along the $\mathrm{z}$-axis to be $v$. The case we discussed above, obtaining the refined MacMahon function, had $v=\emptyset$. For non-trivial $v$ the assignment of $q_{2}$ and $q_{1}$ to various slices is different and depends on the shape of $v$. As we go from $+\infty$ to $-\infty$ the slices are counted with $q_{1}$ if we go towards the left and are counted with $q_{2}$ if we move up. An example is shown in Fig. 4.

After taking into account the framing and the fact that the slices relevant for the topological vertex are not the perpendicular slices [30] the generating function is given by

$$
G_{\lambda \mu \nu}\left(q_{1}, q_{2}\right)=M\left(q_{1}, q_{2}\right) \times C_{\lambda \mu \nu}\left(q_{1}, q_{2}\right)
$$


where $C_{\lambda \mu \nu}\left(q_{1}, q_{2}\right)$ is the refined topological vertex,

$$
\begin{aligned}
C_{\lambda \mu \nu}\left(q_{1}, q_{2}\right)= & \left(\frac{q_{2}}{q_{1}}\right)^{\frac{\|v\|^{2}-\|\nu\|^{2}}{2}} q_{2}^{\frac{\kappa(\mu)}{2}} P_{\nu^{t}}\left(q_{1}^{-\rho} ; q_{2}, q_{1}\right) \sum_{\eta}\left(\frac{q_{2}}{q_{1}}\right)^{\frac{|\eta|+|\lambda|-|\mu|}{2}} \\
& \times s_{\lambda^{t} / \eta}\left(q_{1}^{-\rho} q_{2}^{-\nu}\right) s_{\mu / \eta}\left(q_{1}^{-v^{t}} q_{2}^{-\rho}\right) .
\end{aligned}
$$

In the above expression $P_{v}\left(\mathbf{x} ; q_{2}, q_{1}\right)$ is the Macdonald function such that

$$
\begin{aligned}
P_{v^{t}}\left(q_{1}^{-\rho} ; q_{2}, q_{1}\right)= & q_{1}^{\frac{\|v\|^{2}}{2}} \widetilde{Z}_{v}\left(q_{1}, q_{2}\right), \\
\widetilde{Z}_{v}\left(q_{1}, q_{2}\right)= & \prod_{(i, j) \in v}\left(1-q_{1}^{a(i, j)+1} q_{2}^{\ell(i, j)}\right)^{-1}, a(i, j)=v_{j}^{t}-i, \\
& \quad(i, j)=v_{i}-j .
\end{aligned}
$$

4.1. Open topological string amplitudes. In this section we will discuss the open string partition function obtained from the topological vertex and its relation with polynomial Hopf link invariants. Recall that the usual topological vertex is given by $[18,30]$

$$
C_{\lambda \mu \nu}\left(q_{1}\right)=q_{1}^{\frac{\kappa(\mu)}{2}} s_{\nu^{t}}\left(q_{1}^{-\rho}\right) \sum_{\eta} s_{\lambda^{t} / \eta}\left(q_{1}^{-\rho-v}\right) s_{\mu / \eta}\left(q_{1}^{-\rho-v^{t}}\right) .
$$

Although written in terms of the Schur and skew-Schur functions in the above equation, it can be rewritten in terms of $\operatorname{sl}(N)$ Hopf link invariants for large $N$ [18],

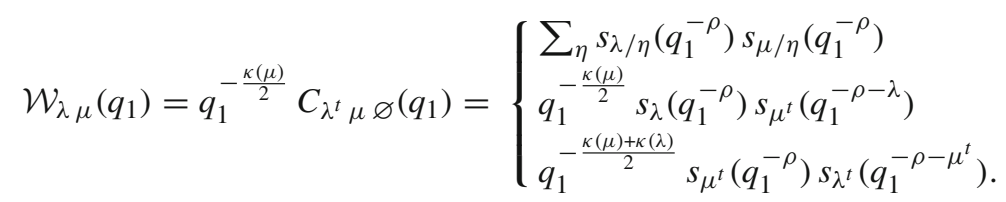

The above three expressions are equivalent because of cyclic symmetry of the topological vertex. Next, we will show that $\operatorname{sl}(N)$ Hopf link invariants can be related to the open string partition function calculated using the topological vertex. Equation (51) will guide us in formulating the precise relation between the $\operatorname{sl}(N)$ Hopf link invariant and the open string partition function.

4.1.1. Hopf link. As we discussed in Sect. 2, after geometric transition, the Hopf link is represented by a pair of toric Lagrangian branes in the geometry $\mathcal{O}(-1) \oplus \mathcal{O}(-1) \mapsto \mathbb{P}^{1}$. Furthermore, as we also discussed earlier, there are two possible resolutions of the singular conifold, both given by $\mathcal{O}(-1) \oplus \mathcal{O}(-1) \mapsto \mathbb{P}^{1}$, related to each other by a flop transition as shown in Fig. 5. We will determine the open string partition function for both these configurations.

The open string partition function for the configuration shown in Fig. 5(a) is given by

$$
Z^{\mathbf{I}}\left(q_{1}, Q, V_{1}, V_{2}\right)=\sum_{\lambda, \mu} Z_{\lambda \mu}^{\mathbf{I}}\left(q_{1}, Q\right) \operatorname{Tr}_{\lambda} V_{1} \operatorname{Tr}_{\mu} V_{2}
$$



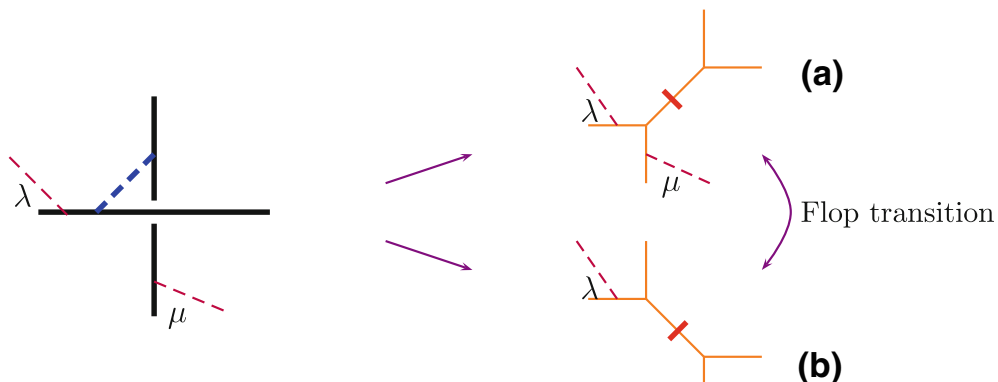

Fig. 5. Two different resolutions of the conifold related to each other by flop transition. The normalized partition function of the geometry (b) gives homological $s l(N)$ invariants of the Hopf link decorated by representations $\left(R_{1}, R_{2}\right)$. The red mark indicates the choice of the preferred direction for the refined vertex

where $V_{1}$ and $V_{2}$ are the two holonomy matrices associated with the two unknot components of the Hopf link and

$$
\begin{aligned}
Z_{\lambda \mu}^{\mathbf{I}}\left(q_{1}, Q\right) & =\sum_{\nu}(-Q)^{|\nu|} C_{\lambda \mu \nu}\left(q_{1}\right) C_{\varnothing \varnothing \nu^{t}}\left(q_{1}\right) \\
& =s_{\lambda^{t}}\left(q_{1}^{-\rho}\right) s_{\mu^{t}}\left(q_{1}^{-\rho-\lambda}, Q q_{1}^{\rho}\right) \prod_{i, j=1}^{\infty}\left(1-Q q_{1}^{i+j-1-\lambda_{j}^{t}}\right) .
\end{aligned}
$$

We normalize the above open string partition function by dividing with the closed string partition function to obtain,

$$
\widehat{Z}_{\lambda \mu}^{\mathbf{I}}\left(q_{1}, Q\right):=\frac{Z_{\lambda \mu}^{\mathbf{I}}\left(q_{1}, Q\right)}{Z_{\varnothing \varnothing}^{\mathbf{I}}\left(q_{1}, Q\right)}=s_{\lambda^{t}}\left(q_{1}^{-\rho}\right) s_{\mu^{t}}\left(q_{1}^{-\rho-\lambda}, Q q_{1}^{\rho}\right) \prod_{(i, j) \in \lambda}\left(1-Q q_{1}^{j-i}\right) .
$$

In the limit $Q \mapsto 0$ we get

$$
\widehat{Z}_{\lambda \mu}^{\mathbf{I}}\left(q_{1}, Q=0\right)=C_{\lambda \mu \varnothing}=q_{1}^{\frac{\kappa(\mu)}{2}} \mathcal{W}_{\lambda^{t} \mu}\left(q_{1}\right) .
$$

The right-hand side is the large $N$ limit of the $\operatorname{sl}(N)$ Hopf link invariant. The above equation suggests the following relation between the open string partition function and the $\operatorname{sl}(N)$ Hopf link invariant:

$$
W_{\lambda \mu}\left(q_{1}, N\right)=q_{1}^{-\frac{\kappa(\mu)}{2}} \widehat{Z}_{\lambda^{t} \mu}^{\mathbf{I}}\left(q_{1}, Q\right), \quad Q=q_{1}^{N} .
$$

For $(\lambda, \mu)=(\square, \square)$ we get

$$
\begin{aligned}
W_{\square \square}\left(q_{1}, N\right)=\widehat{Z}_{\square \square}^{\mathbf{I}}\left(q_{1}, Q\right) & =s_{\square}\left(q_{1}^{-\rho-\square}, Q q_{1}^{\rho}\right) q_{1}^{\frac{1}{2}} \frac{1-Q}{1-q_{1}} \\
& =\left(q_{1}^{-\frac{1}{2}}+\frac{q_{1}^{\frac{3}{2}}}{1-q_{1}}-Q \frac{q_{1}^{\frac{1}{2}}}{1-q_{1}}\right) q_{1}^{\frac{1}{2}} \frac{1-Q}{1-q_{1}} \\
& =\frac{1-q_{1}+q_{1}^{2}}{\left(1-q_{1}\right)^{2}}-Q \frac{1+q_{1}^{2}}{\left(1-q_{1}\right)^{2}}+Q^{2} \frac{q_{1}}{\left(1-q_{1}\right)^{2}} .
\end{aligned}
$$


Flop transition. The other possibility for the geometry after transition is as shown in Fig. 5(b). In this case the partition function is given by

$$
\begin{aligned}
Z_{\lambda \mu}^{\mathrm{II}}\left(q_{1}, \widehat{Q}\right) & =\sum_{\nu}(-\widehat{Q})^{|\nu|} C_{\varnothing \mu \nu}\left(q_{1}\right) C_{\lambda \varnothing \nu^{t}}\left(q_{1}\right) \\
& =q_{1}^{\frac{\kappa(\mu)}{2}} \sum_{\nu}(-\widehat{Q})^{|v|} s_{\nu}\left(q_{1}^{-\rho}\right) s_{\nu^{t}}\left(q_{1}^{-\rho}\right) s_{\lambda^{t}}\left(q_{1}^{-\rho-v^{t}}\right) s_{\mu}\left(q_{1}^{-\rho-v^{t}}\right) .
\end{aligned}
$$

For $(\lambda, \mu)=(\square, \square)$ we get

$$
\begin{aligned}
\widehat{Z}_{\square \square}^{\text {II }}\left(q_{1}, \widehat{Q}\right) & =\frac{Z_{\square \square}^{\text {II }}\left(q_{1}, \widehat{Q}\right)}{Z_{\varnothing \varnothing}^{\text {II }}\left(q_{1}, \widehat{Q}\right)} \\
& =\frac{q_{1}}{\left(1-q_{1}\right)^{2}}-\widehat{Q} \frac{1+q_{1}^{2}}{\left(1-q_{1}\right)^{2}}+\widehat{Q}^{2} \frac{1-q_{1}+q_{1}^{2}}{\left(1-q_{1}\right)^{2}} \\
& =\widehat{Q}^{2}\left[\frac{1-q_{1}+q_{1}^{2}}{\left(1-q_{1}\right)^{2}}-\widehat{Q}^{-1} \frac{1+q_{1}^{2}}{\left(1-q_{1}\right)^{2}}+\widehat{Q}^{-2} \frac{q_{1}}{\left(1-q_{1}\right)^{2}}\right] \\
& =\widehat{Q}^{2} \widehat{Z}_{\square \square}^{\mathbf{I}}\left(q_{1}, \widehat{Q}^{-1}\right) .
\end{aligned}
$$

Thus we see that the two partition functions are equal (up to an overall factor) if we define the Kähler parameters for these two cases, related by the flop transition, as

$$
\widehat{Q}=Q^{-1} \text {. }
$$

This implies that

$$
W_{\lambda \mu}\left(q_{1}, N\right)=q_{1}^{-\frac{\kappa(\mu)}{2}}\left(Q^{-1}\right)^{\frac{|\lambda|+|\mu|}{2}} \widehat{Z}_{\lambda^{t} \mu}^{\text {II }}\left(q_{1}, Q\right), \quad Q=q_{1}^{-N} .
$$

Thus we see that when using the usual topological vertex we get the same result for the two geometries (with branes) related by flop transition. This "symmetry", however, is not preserved by the refined topological vertex as we will see in the next section.

\section{Refined Vertex and Link Homologies}

In this section we will determine the refined open topological string partition functions for the two configuration of branes on the resolved conifold shown in Fig. 5. Let us begin by defining the refined topological vertex that we will use:

$$
\begin{aligned}
C_{\lambda \mu \nu}\left(q_{1}, q_{2}\right)= & \left(\frac{q_{1}}{q_{2}}\right)^{\frac{\left\|\mu\left|\|^{2}-\right| \mu \mid\right.}{2}} q_{2}^{\frac{\kappa(\mu)}{2}} q_{2}^{\frac{\|v\|^{2}}{2}} \widetilde{Z}_{v}\left(q_{1}, q_{2}\right) \\
& \times \sum_{\eta}\left(\frac{q_{2}}{q_{1}}\right)^{\frac{|\eta|+|\lambda|-|\mu|}{2}} s_{\lambda^{t} / \eta}\left(q_{1}^{-\rho} q_{2}^{-v}\right) s_{\mu / \eta}\left(q_{2}^{-\rho} q_{1}^{-v^{t}}\right) .
\end{aligned}
$$

The above definition of the refined topological vertex differs from the refined vertex in [17] by a factor which does not affect the closed string calculations because it cancels due to interchanging of $q_{1}, q_{2}$ in gluing the vertex along an internal line. For the open 
string partition functions this factor only appears as an overall factor multiplying the partition function. by

The open string refined partition function of the geometry shown in Fig. 5(b) is given

$$
Z_{\lambda \mu}\left(q_{1}, q_{2}, Q\right)=\sum_{\nu}(-Q)^{|\nu|} C_{\varnothing \mu \nu}\left(q_{1}, q_{2}\right) C_{\lambda \varnothing \nu^{t}}\left(q_{2}, q_{1}\right)
$$

Since

$$
\begin{aligned}
& C_{\varnothing \mu \nu}\left(q_{1}, q_{2}\right)=\left(\frac{q_{1}}{q_{2}}\right)^{\frac{\|\mu\|^{2}}{2}} q_{2}^{\frac{\kappa(\mu)}{2}} q_{2}^{\frac{\|v\|^{2}}{2}} \widetilde{Z}_{\nu}\left(q_{1}, q_{2}\right) s_{\mu}\left(q_{2}^{-\rho} q_{1}^{-v^{t}}\right), \\
& C_{\lambda \varnothing \nu^{t}}\left(q_{2}, q_{1}\right)=\left(\frac{q_{2}}{q_{1}}\right)^{\frac{|\lambda|}{2}} q_{1}^{\frac{\left\|v^{t}\right\|^{2}}{2}} \widetilde{Z}_{\nu^{t}}\left(q_{2}, q_{1}\right) s_{\lambda^{t}}\left(q_{2}^{-\rho} q_{1}^{-v^{t}}\right),
\end{aligned}
$$

the open string partition function becomes

$$
\begin{aligned}
Z_{\lambda \mu}\left(q_{1}, q_{2}, Q\right)= & h_{\lambda \mu}\left(q_{1}, q_{2}\right) \sum_{\nu}(-Q)^{|v|} q_{2}^{\frac{\|v\|^{2}}{2}} q_{1}^{\frac{\left\|v^{t}\right\|^{2}}{2}} \widetilde{Z}_{\nu}\left(q_{1}, q_{2}\right) \widetilde{Z}_{\nu^{t}}\left(q_{2}, q_{1}\right) \\
& \times s_{\lambda^{t}}\left(q_{2}^{-\rho} q_{1}^{-v^{t}}\right) s_{\mu}\left(q_{2}^{-\rho} q_{1}^{-v^{t}}\right) \\
h_{\lambda \mu}\left(q_{1}, q_{2}\right)= & \left(\frac{q_{1}}{q_{2}}\right)^{\frac{\left.\|\mu\|\right|^{2}}{2}-\frac{|\lambda|}{2}} q_{2}^{\frac{\kappa(\mu)}{2}} .
\end{aligned}
$$

The normalized partition function is given by

$$
\widehat{Z}_{\lambda \mu}\left(q_{1}, q_{2}, Q\right)=\frac{Z_{\lambda \mu}\left(q_{1}, q_{2}, Q\right)}{Z_{\varnothing \varnothing\left(q_{1}, q_{2}, Q\right)}},
$$

where

$$
\begin{aligned}
Z_{\varnothing \varnothing\left(q_{1}, q_{2}, Q\right)} & =\sum_{\nu}(-Q)^{|v|} q_{2}^{\frac{\|v\|^{2}}{2}} q_{1}^{\frac{\left\|v^{t}\right\|^{2}}{2}} \widetilde{Z}_{v}\left(q_{1}, q_{2}\right) \widetilde{Z}_{v^{t}}\left(q_{2}, q_{1}\right) \\
& =\prod_{i, j=1}^{\infty}\left(1-Q q_{1}^{i-\frac{1}{2}} q_{2}^{j-\frac{1}{2}}\right) .
\end{aligned}
$$

Recall that the $\operatorname{sl}(N)$ Hopf link invariant is related to the open string partition function as

$$
W_{\lambda \mu}(q, N)=q^{-\frac{\kappa(\mu)}{2}}\left(Q^{-1}\right)^{\frac{|\lambda|+|\mu|}{2}} \widehat{Z}_{\lambda^{t} \mu}^{\text {II }}\left(q, Q=q^{-N}\right)
$$

The factor $q^{-\frac{\kappa(\mu)}{2}}$ is the framing factor for the usual topological vertex. For the case of the refined vertex the framing factor is given by [17]

$$
f_{\lambda}\left(q_{1}, q_{2}\right)=\left(\frac{q_{2}}{q_{1}}\right)^{\frac{\| \mu^{t}||^{2}-|\mu|}{2}} q_{1}^{-\frac{\kappa(\mu)}{2}} .
$$


Therefore we conjecture the following relation between the homological $s l(N)$ invariants of the Hopf link and the refined open string partition function ${ }^{5}$ :

$$
\begin{aligned}
& \overline{\mathcal{P}}_{\lambda \mu}(q, t, \mathbf{a})=(-1)^{|\lambda|+|\mu|}\left(\frac{q_{1}}{q_{2}}\right)^{|\lambda|+|\lambda||\mu|} f_{\lambda}\left(q_{1}, q_{2}\right) \\
& \times\left(Q^{-1} \sqrt{\frac{q_{1}}{q_{2}}}\right)^{\frac{|\lambda|+|\mu|}{2}} \widehat{Z}_{\lambda^{t} \mu}^{\text {II }}\left(q_{1}, q_{2}, Q\right) \\
& =\left[\sum_{v}(-Q)^{|v|} q_{2}^{\frac{\|v\|^{2}}{2}} q_{1}^{\frac{\left\|v^{t}\right\|^{2}}{2}}\right. \\
& \left.\times \widetilde{Z}_{v}\left(q_{1}, q_{2}\right) \widetilde{Z}_{v^{t}}\left(q_{2}, q_{1}\right) s_{\lambda}\left(q_{2}^{-\rho} q_{1}^{-v^{t}}\right) s_{\mu}\left(q_{2}^{-\rho} q_{1}^{-v^{t}}\right)\right]
\end{aligned}
$$

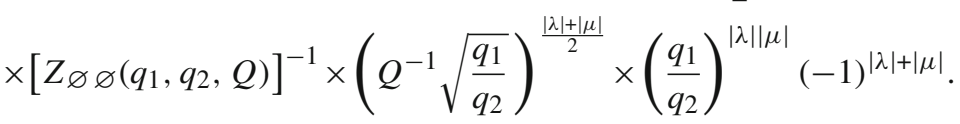

This is one of the main results of the present paper. The map between the knot theory parameters $(q, t, \mathbf{a})$ and the vertex parameters $\left(q_{1}, q_{2}, Q\right)$ is given by $(5)$, where $\mathbf{a}=q^{N}$, and the limit in which we recover the usual topological vertex calculation is given by $t=-1$.

5.1. Unknot. From now on we will drop the superscript II on the normalized partition function and will just write it as $\widehat{Z}_{\lambda \mu}\left(q_{1}, q_{2}, Q\right)$. Below we compute the Poincaré polynomial (67) of the triply-graded homology for small representations $(\lambda, \mu)$ and compare with known results, whenever they are available.

For the case $(\lambda, \mu)=(\square, \varnothing)$ we get

$$
\begin{aligned}
\overline{\mathcal{P}}_{\square \varnothing(t, q, \mathbf{a})} & =-\mathbf{a} \frac{\sum_{\nu}(-Q)^{|\nu|} q_{2}^{\frac{\|v\|^{2}}{2}} q_{1}^{\frac{\|v\|^{2}}{2}} \widetilde{Z}_{\nu}\left(q_{1}, q_{2}\right) \widetilde{Z}_{v^{t}}\left(q_{2}, q_{1}\right) s_{\square}\left(q_{2}^{-\rho} q_{1}^{-v^{t}}\right)}{\prod_{i, j=1}^{\infty}\left(1-Q q_{1}^{i-\frac{1}{2}} q_{2}^{j-\frac{1}{2}}\right)} \\
& =-\mathbf{a}\left(\frac{\sqrt{q_{2}}}{1-q_{2}}-Q \sqrt{\frac{q_{2}}{q_{1}}} \frac{\sqrt{q_{2}}}{1-q_{2}}\right)=\mathbf{a}\left(\frac{1}{q-q^{-1}}-\frac{\mathbf{a}^{-2}}{q-q^{-1}}\right) \\
& =\frac{\mathbf{a}-\mathbf{a}^{-1}}{q-q^{-1}}, \quad \mathbf{a}=q^{N},
\end{aligned}
$$

which is exactly the superpolynomial of the unknot [9].

It is interesting to note that for generic representations the partition function for the unknot depends on both parameters $q$ and $t$, whose interpretation we are currently investigating [31]. However, for totally anti-symmetric representations it is expected to be only a function of $q$ given by (3). Indeed, for $\theta=\Lambda^{2}$ and $\theta=\Lambda^{3}$ we find:

5 The factor $\left(\frac{q_{1}}{q_{2}}\right)^{|\lambda|}$ has been introduced to make the expression symmetric in $\lambda$ and $\mu$. 


$$
\begin{aligned}
\overline{\mathcal{P}}_{\Lambda^{2}}(t, q, \mathbf{a})= & \mathbf{a}^{2}\left(\frac{q^{4}}{\left(1-q^{2}\right)\left(1-q^{4}\right)}-\frac{\mathbf{a}^{-2} q^{4}}{\left(1-q^{2}\right)^{2}}+\frac{\mathbf{a}^{-4} q^{6}}{\left(1-q^{2}\right)\left(1-q^{4}\right)}\right) \\
\overline{\mathcal{P}}_{\Lambda^{3}}(t, q, \mathbf{a})= & \mathbf{a}^{3}\left(-\frac{q^{9}}{\left(1-q^{2}\right)^{3}\left(1+2 q^{2}+2 q^{4}+q^{6}\right)}+\frac{\mathbf{a}^{-2} q^{9}}{\left(1-q^{2}\right)^{2}\left(1-q^{4}\right)}\right. \\
& \left.-\frac{\mathbf{a}^{-4} q^{11}}{\left(1-q^{2}\right)^{2}\left(1-q^{4}\right)}+\frac{\mathbf{a}^{-6} q^{15}}{\left(1-q^{2}\right)^{3}\left(1+2 q^{2}+2 q^{4}+q^{6}\right)}\right)
\end{aligned}
$$

in complete agreement with (3). Note that for $\mathbf{a}=q^{N}$ the partition functions reduce to finite polynomials in $q$ with non-negative integer coefficients.

For representations other than the antisymmetric ones the refined partition function (67) depends on $t$ in a non-trivial way.

5.2. Hopf link. Let us now consider the Hopf link colored by $\left(R_{1}, R_{2}\right)=(\square, \square)$. In this case, from Eqs. (5) and (67) we get

$$
\begin{aligned}
& \overline{\mathcal{P}}_{\square \square}(t, q, \mathbf{a}) \\
& =Q^{-1} \sqrt{\frac{q_{1}}{q_{2}}} \frac{\left(\frac{q_{1}}{q_{2}}\right) \sum_{\nu}(-Q)^{|v|} q_{2}^{\frac{\|v\|^{2}}{2}} q_{1}^{\frac{\left\|v^{t}\right\|^{2}}{2}} \widetilde{Z}_{v}\left(q_{1}, q_{2}\right) \widetilde{Z}_{v^{t}}\left(q_{2}, q_{1}\right)\left(s_{\square}\left(q_{2}^{-\rho} q_{1}^{-v^{t}}\right)\right)^{2}}{\prod_{i, j=1}^{\infty}\left(1-Q q_{1}^{i-\frac{1}{2}} q_{2}^{j-\frac{1}{2}}\right)} \\
& =\mathbf{a}^{2}\left(\frac{q_{1}}{\left(1-q_{2}\right)^{2}}-Q\left(\frac{q_{2}}{q_{1}}\right)^{\frac{1}{2}} \frac{1+q_{1}-q_{2}+q_{1} q_{2}}{\left(1-q_{2}\right)^{2}}+Q^{2}\left(\frac{q_{2}}{q_{1}}\right) \frac{1-q_{2}+q_{1} q_{2}}{\left(1-q_{2}\right)^{2}}\right) \\
& =\mathbf{a}^{2}\left(\frac{q_{1}}{\left(1-q_{2}\right)^{2}}-\mathbf{a}^{-2} \frac{1+q_{1}-q_{2}+q_{1} q_{2}}{\left(1-q_{2}\right)^{2}}+\mathbf{a}^{-4} \frac{1-q_{2}+q_{1} q_{2}}{\left(1-q_{2}\right)^{2}}\right) \\
& =\mathbf{a}^{-2}\left(\frac{1-q^{2}+q^{4} t^{2}}{\left(1-q^{2}\right)^{2}}-\mathbf{a}^{2} \frac{1+q^{2} t^{2}-q^{2}+q^{4} t^{2}}{\left(1-q^{2}\right)^{2}}+\mathbf{a}^{4} \frac{q^{2} t^{2}}{\left(1-q^{2}\right)^{2}}\right) .
\end{aligned}
$$

This result agrees with the superpolynomial of the Hopf link computed in Eq. (38).

For $\mathbf{a}=q^{N}$ we get

$$
\begin{aligned}
& \overline{\mathcal{P}}_{\square} \square\left(q, t, \mathbf{a}=q^{N}\right) \\
& =q^{-2 N}\left\{\frac{1-q^{2}+q^{4} t^{2}}{\left(1-q^{2}\right)^{2}}-q^{2 N} \frac{1+q^{2} t^{2}-q^{2}+q^{4} t^{2}}{\left(1-q^{2}\right)^{2}}+q^{4 N} \frac{q^{2} t^{2}}{\left(1-q^{2}\right)^{2}}\right\} \\
& =q^{-2 N}\left\{\frac{\left(1-q^{2}\right)\left(1-q^{2 N}\right)}{\left(1-q^{2}\right)^{2}}+t^{2}\left(\frac{q^{4}-q^{2 N+2}-q^{2 N+4}+q^{4 N+2}}{\left(1-q^{2}\right)^{2}}\right)\right\} \\
& =q^{-2 N}\left\{q^{N-1}\left(\frac{q^{N}-q^{-N}}{q-q^{-1}}\right)+t^{2}\left(\frac{q^{2}-q^{2 N}-q^{2 N+2}+q^{4 N}}{\left(q-q^{-1}\right)^{2}}\right)\right\} \\
& =q^{-2 N}\left\{q^{N-1}\left(\frac{q^{N}-q^{-N}}{q-q^{-1}}\right)+t^{2}\left(\frac{\left(1-q^{2 N}\right)^{2}-\left(1-q^{2}\right)\left(1-q^{2 N}\right)}{\left(q-q^{-1}\right)^{2}}\right)\right\} \\
& =q^{-2 N}\left\{q^{N-1}\left(\frac{q^{N}-q^{-N}}{q-q^{-1}}\right)+t^{2}\left(\frac{1-q^{2 N}}{q-q^{-1}}\right)^{2}+t^{2} q \frac{1-q^{2 N}}{q-q^{-1}}\right\}
\end{aligned}
$$




$$
\begin{aligned}
& =q^{-2 N}\left\{q^{N-1}\left(\frac{q^{N}-q^{-N}}{q-q^{-1}}\right)+t^{2} q^{2 N}\left(\frac{q^{N}-q^{-N}}{q-q^{-1}}\right)^{2}-t^{2} q^{N+1}\left(\frac{q^{N}-q^{-N}}{q-q^{-1}}\right)\right\} \\
& =q^{-2 N} K h_{N}\left(2_{1}^{2}\right),
\end{aligned}
$$

which is exactly the expression Eq. (39) calculated in Sect. 3.

Hopf link colored by ( $\square, \boxminus)$. For the Hopf link colored by ( $\square, \boxminus$ ) we get

$$
\begin{aligned}
\overline{\mathcal{P}}_{(\square, 日)}(t, q, \mathbf{a})= & \frac{\mathbf{a}^{-3}\left(1-q^{4}+q^{6} t^{2}\right)}{\left(1-q^{2}\right)^{2}\left(1-q^{4}\right)} \\
& -\frac{\mathbf{a}^{-1} q^{-2}\left(1+q^{2}-q^{4}-q^{6}+q^{4} t^{2}+q^{6} t^{2}+q^{8} t^{2}\right)}{\left(1-q^{2}\right)^{2}\left(1-q^{4}\right)} \\
& +\frac{\mathbf{a} q^{-2}\left(1-q^{4}+q t^{2}+q^{4} t^{2}+q^{6} t^{2}\right)}{\left(1-q^{2}\right)^{2}\left(1-q^{4}\right)}-\frac{\mathbf{a}^{3} t^{2}}{\left(1-q^{2}\right)^{2}\left(1-q^{4}\right)} .
\end{aligned}
$$

There is no knot theory result with which we can compare this result. However, note that this has all the right properties. It vanishes for $\mathbf{a}=1$, i.e., $N=0$ and for $\mathbf{a}=q^{N}$ it gives $q^{-3 N}$ times a finite polynomial with positive integer coefficients:

$$
\begin{aligned}
\overline{\mathcal{P}}_{(\square, 日)}(t, q, \mathbf{a}=1)= & \overline{\mathcal{P}}_{(\square, \boxminus)}(t, q, \mathbf{a}=q)=0, \\
\overline{\mathcal{P}}_{(\square, \boxminus)}\left(t, q, \mathbf{a}=q^{2}\right)= & q^{-6}\left(1+q^{2}\right), \\
\overline{\mathcal{P}}_{(\square, 日)}\left(t, q, \mathbf{a}=q^{3}\right)= & q^{-9}\left(1+2 q^{2}+2 q^{4}+q^{6}+t^{2} q^{6}+t^{2} q^{8}+t^{2} q^{10}\right), \\
\overline{\mathcal{P}}_{(\square, 日)}\left(t, q, \mathbf{a}=q^{4}\right)= & q^{-12}\left(1+2 q^{2}+3 q^{4}+3 q^{6}+2 q^{8}+q^{10}\right. \\
& \left.+t^{2} q^{6}\left(1+2 q^{2}+3 q^{4}+3 q^{6}+2 q^{8}+q^{10}\right)\right), \\
\overline{\mathcal{P}}_{(\square, 日)}\left(t, q, \mathbf{a}=q^{5}\right)= & q^{-15}\left(1+2 q^{2}+3 q^{4}+\left(4+t^{2}\right) q^{6}+\left(4+2 t^{2}\right) q^{8}\right. \\
& +\left(3+4 t^{2}\right) q^{10}+\left(2+5 t^{2}\right) q^{12}+\left(1+6 t^{2}\right) q^{14}+5 t^{2} q^{16} \\
& \left.+4 t^{2} q^{18}+2 t^{2} q^{20}+t^{2} q^{22}\right), \\
\overline{\mathcal{P}}_{(\square, 日)}\left(t, q, \mathbf{a}=q^{6}\right)= & q^{-18}\left(1+2 q^{2}+3 q^{4}+\left(4+t^{2}\right) q^{6}+\left(5+2 t^{2}\right) q^{8}\right. \\
& +\left(5+4 t^{2}\right) q^{10}+\left(4+6 t^{2}\right) q^{12} \\
& +\left(3+8 t^{2}\right) q^{14}+\left(2+9 t^{2}\right) q^{16}+\left(1+9 t^{2}\right) q^{18}+8 t^{2} q^{20} \\
& \left.+6 t^{2} q^{22}+4 t^{2} q^{24}+2 t^{2} q^{26}+t^{2} q^{28}\right) .
\end{aligned}
$$

Acknowledgements. We would like to thank C. Doran, J. Rasmussen, and B. Webster for valuable discussions. It is our pleasure to thank the Stony Brook physics department and the $4^{\text {th }}$ Simons Workshop in Mathematics and Physics for hospitality during the initial stages of this work. In addition, C.V. thanks the CTP at MIT for hospitality during his sabbatical leave. The work of S.G. is supported in part by DOE grant DE-FG03-92-ER40701, in part by RFBR grant 04-02-16880, and in part by the grant for support of scientific schools NSh-8004.2006.2. The work of C.V. is supported in part by NSF grants PHY-0244821 and DMS-0244464.

Open Access This article is distributed under the terms of the Creative Commons Attribution Noncommercial License which permits any noncommercial use, distribution, and reproduction in any medium, provided the original author(s) and source are credited. 


\section{A. Appendix: Other Representations}

In this appendix we write the normalized partition function of the Hopf link and unknot colored by other representations of $s l(N)$ which, as usual, we label by partitions (or Young diagrams). Specifically, we list simple examples where Young diagrams have at most two columns.

Let us define

$$
\begin{aligned}
& G_{\lambda \mu}(-Q, q, t) \\
& :=\left[\sum_{\nu}(-Q)^{|\nu|} q_{2}^{\frac{\|v\|^{2}}{2}} q_{1}^{\frac{\left\|v^{t}\right\|^{2}}{2}} \widetilde{Z}_{\nu}\left(q_{1}, q_{2}\right) \widetilde{Z}_{\nu^{t}}\left(q_{2}, q_{1}\right) s_{\lambda}\left(q_{2}^{-\rho} q_{1}^{-v^{t}}\right) s_{\mu}\left(q_{2}^{-\rho} q_{1}^{-v^{t}}\right)\right]
\end{aligned}
$$

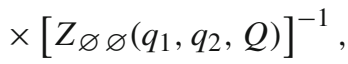

where we used the identification $\left(q_{1}, q_{2}\right)=\left(t^{2} q^{2}, q^{2}\right)$ to write $G$ as a function of $q$ and $t$. In terms of $G_{\lambda \mu}(Q, q, t)$ the normalized partition function is given by

$$
\begin{aligned}
\widehat{Z}_{\lambda \mu}\left(q_{1}, q_{2}, Q\right) & =h_{\lambda \mu} G_{\lambda^{t} \mu}(-Q, q, t), \\
h_{\lambda \mu} & =q^{\kappa(\mu)} t^{\| \mu||^{2}-|\lambda|} .
\end{aligned}
$$

In the next two sections we list $G_{\lambda \mu}$ for various Young diagrams.

\section{A.1. Unknot.}

$$
\begin{aligned}
& G_{(1)}(Q, q, t)=\frac{q}{1-q^{2}}+\frac{\left(\frac{q}{t}\right) Q}{1-q^{2}} \\
& G_{\left(1^{2}\right)}(Q, q, t)=\frac{q^{4}}{\left(1-q^{2}\right)\left(1-q^{4}\right)}+\frac{\left(\frac{q^{4}}{t}\right) Q}{\left(1-q^{2}\right)^{2}}+\frac{\left(\frac{q^{6}}{t^{2}}\right) Q^{2}}{\left(1-q^{2}\right)\left(1-q^{4}\right)} \\
& G_{(2)}(Q, q, t)=\frac{q^{2}}{\left(1-q^{2}\right)\left(1-q^{4}\right)}+\frac{\left(1-q^{2}+q^{2} t^{2}\right) Q}{t^{3}\left(1-q^{2}\right)^{2}}+\frac{\left(1-q^{4}+q^{4} t^{2}\right) Q^{2}}{t^{4}\left(1-q^{2}\right)\left(1-q^{4}\right)}
\end{aligned}
$$

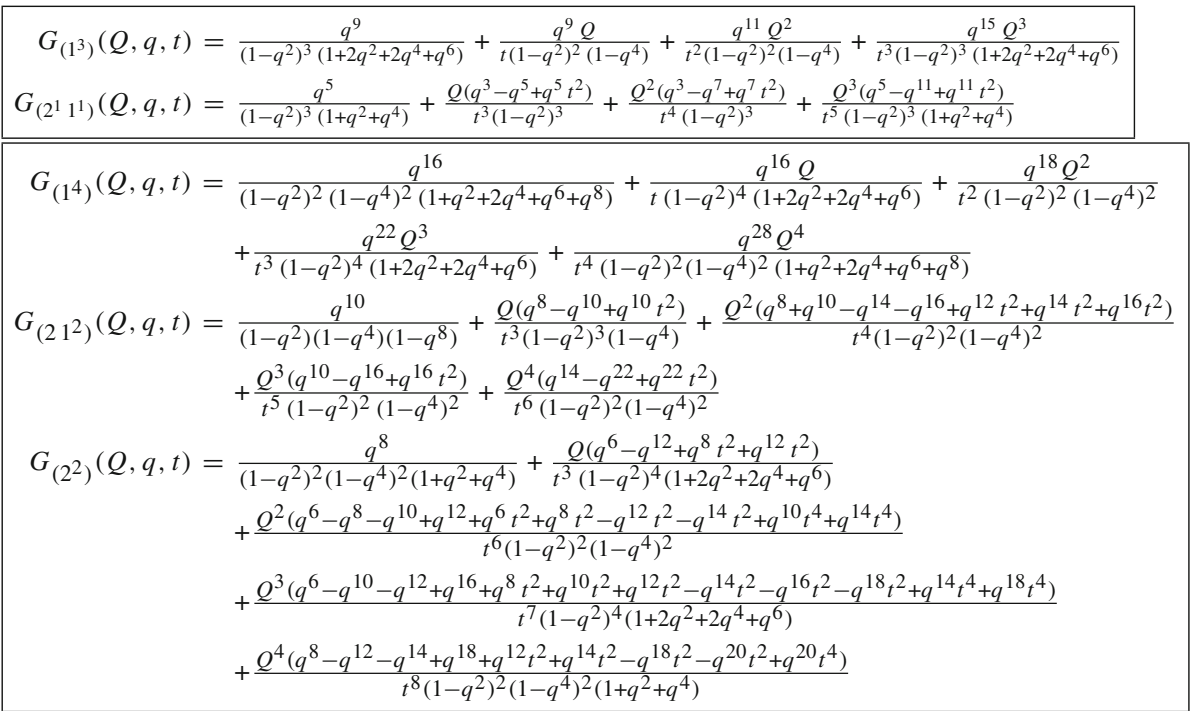




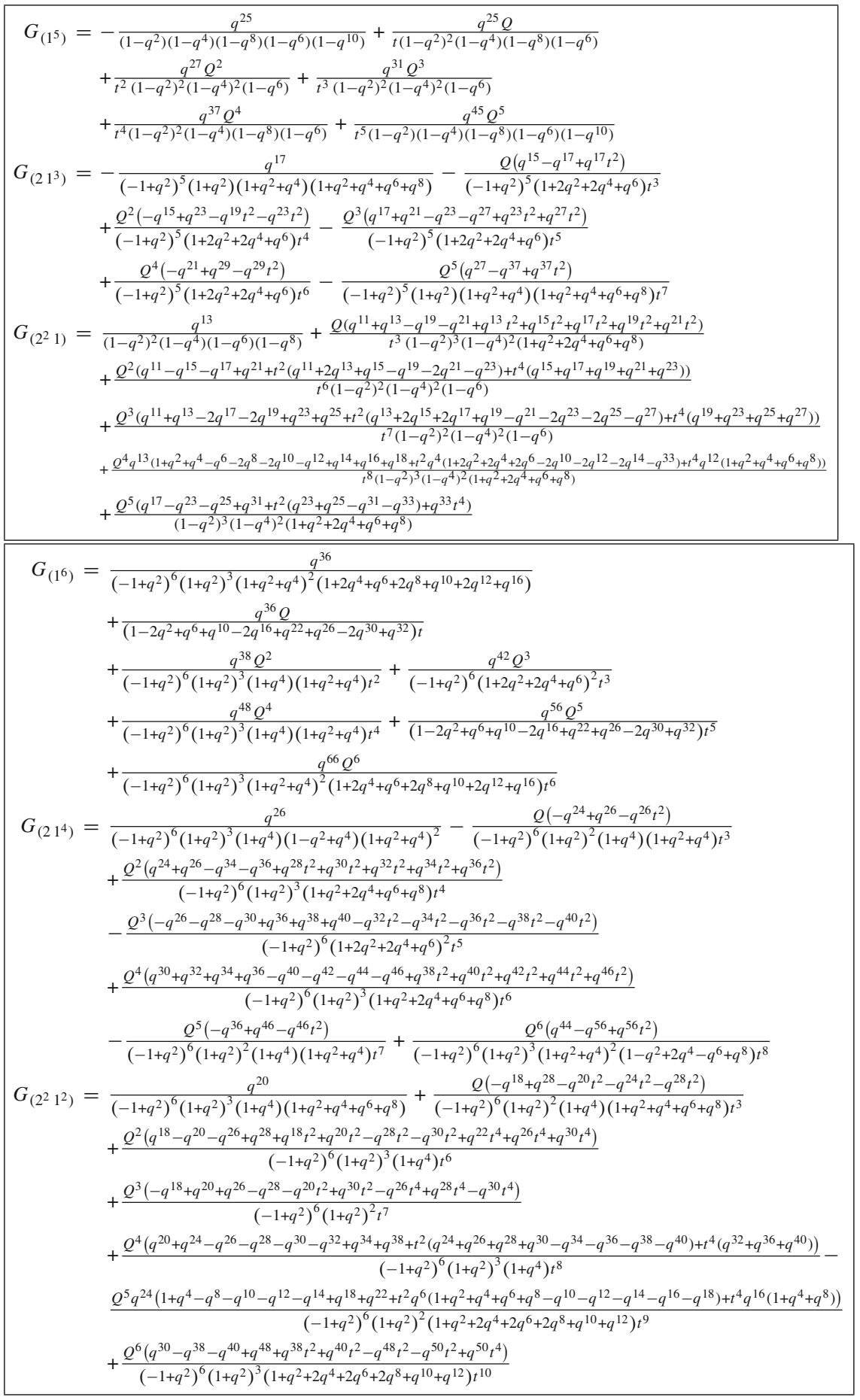




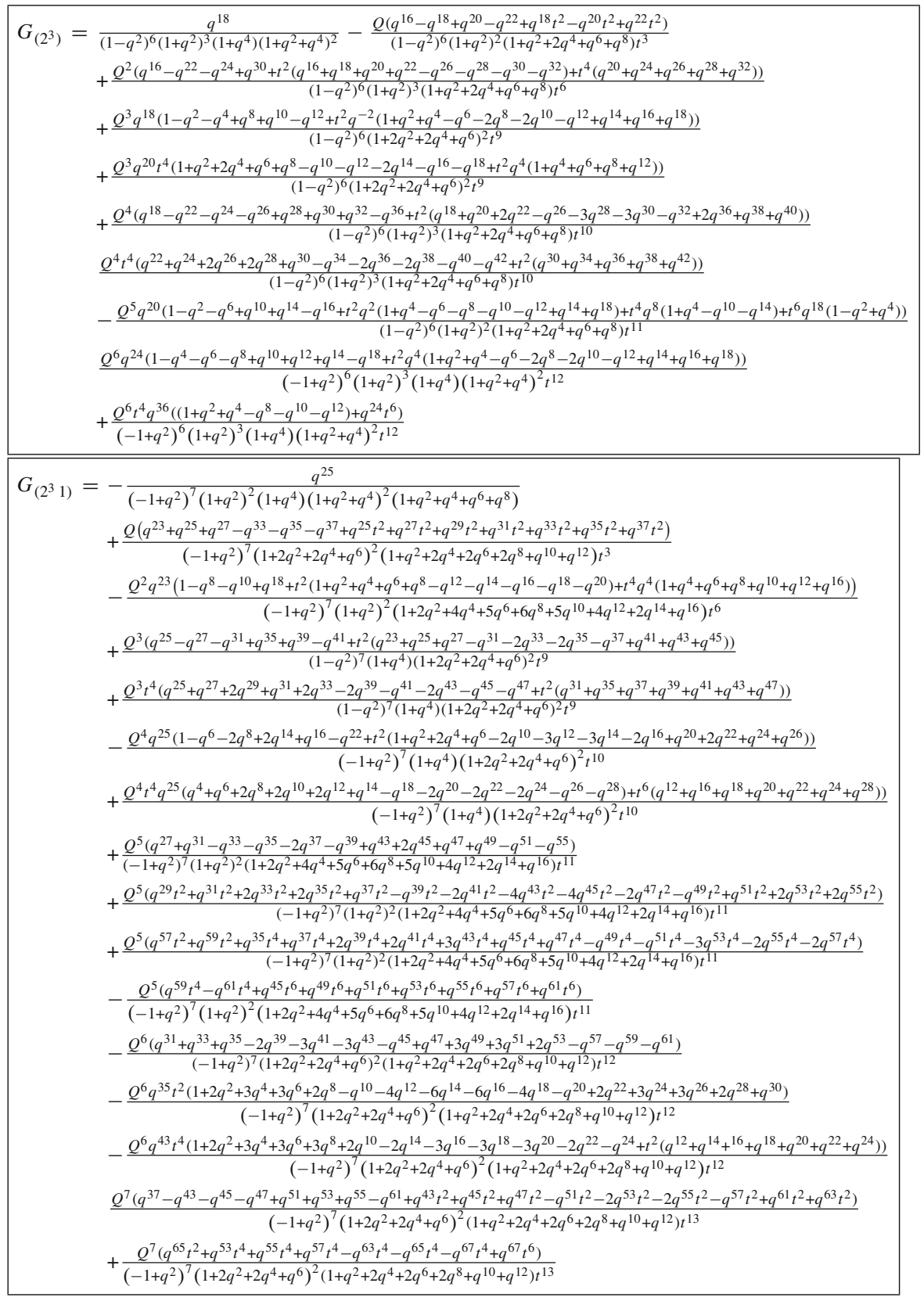

\section{A.2. Hopf link.}

$G_{(1)(1)}=\frac{q^{2}}{\left(1-q^{2}\right)^{2}}-\frac{Q\left(1-q^{2}+q^{2} t^{2}+q^{4} t^{2}\right)}{t^{3}\left(1-q^{2}\right)^{2}}+\frac{Q^{2}\left(1-q^{2}+q^{4} t^{2}\right)}{t^{4}\left(1-q^{2}\right)^{2}}$

$G_{(1)\left(1^{2}\right)}=\frac{q^{5}}{\left(1-q^{2}\right)^{2}\left(1-q^{4}\right)}-\frac{Q\left(q^{3}-q^{7}+q^{5} t^{2}+q^{7} t^{2}+q^{9} t^{2}\right)}{t^{3}\left(1-q^{2}\right)^{2}\left(1-q^{4}\right)}+\frac{Q^{2}\left(q^{3}+q^{5}-q^{7}-q^{9}+q^{7} t^{2}+q^{9} t^{2}+q^{11} t^{2}\right)}{t^{4}\left(1-q^{2}\right)^{2}\left(1-q^{4}\right)}-\frac{Q^{3}\left(q^{5}-q^{9}+q^{11} t^{2}\right)}{t^{5}\left(1-q^{2}\right)^{2}\left(1-q^{4}\right)}$ 


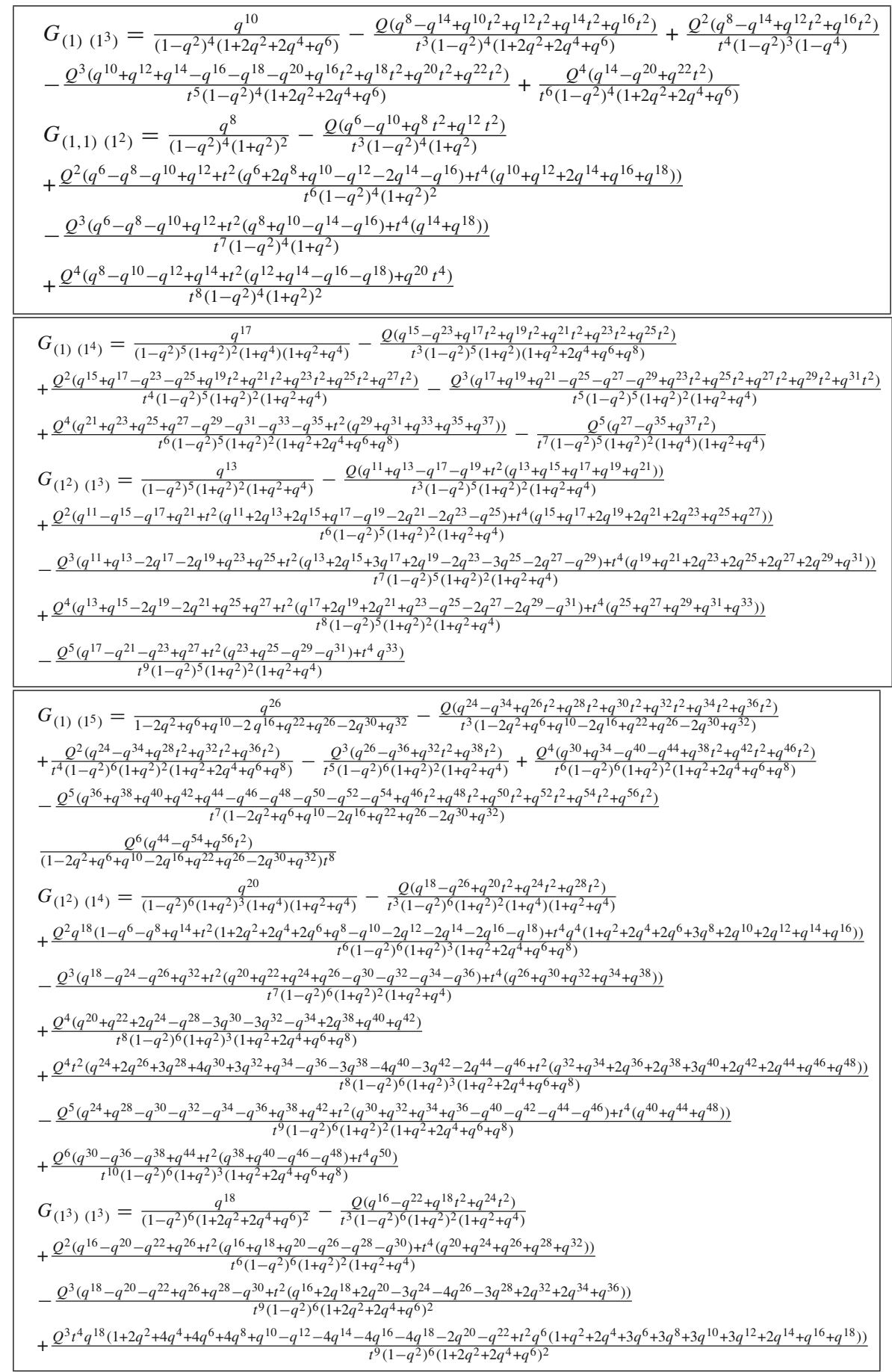


$+\frac{Q^{4}\left(q^{18}-q^{20}-q^{22}+q^{26}+q^{28}-q^{30}+t^{2}\left(q^{18}+q^{20}+q^{22}-q^{24}-2 q^{26}-2 q^{28}-q^{30}+q^{32}+q^{34}+q^{36}\right)\right)}{t^{10}\left(1-q^{2}\right)^{6}\left(1+q^{2}\right)^{2}\left(1+q^{2}+q^{4}\right)}$
$+\frac{Q^{4} t^{4}\left(q^{22}+q^{24}+2 q^{26}+q^{28}+q^{30}-q^{32}-q^{34}-3 q^{36}-q^{38}-q^{40}+t^{2}\left(q^{30}+q^{34}+q^{36}+q^{38}+q^{42}\right)\right)}{t^{10}\left(1-q^{2}\right)^{6}\left(1+q^{2}\right)^{2}\left(1+q^{2}+q^{4}\right)}$
$-\frac{Q^{5}\left(q^{20}-q^{22}-q^{24}+q^{28}+q^{30}-q^{32}+t^{2}\left(q^{22}+q^{24}-q^{28}-2 q^{30}-q^{32}+q^{36}+q^{38}\right)+t^{4}\left(q^{28}+q^{30}+q^{32}-q^{38}-q^{40}-q^{42}\right)+t^{6}\left(q^{38}+q^{44}\right)\right)}{t^{11}\left(1-q^{2}\right)^{6}\left(1+q^{2}\right)^{2}\left(1+q^{2}+q^{4}\right)}$
$+\frac{Q^{6}\left(q^{24}-q^{26}-q^{28}+q^{32}+q^{34}-q^{36}+t^{2}\left(q^{28}+q^{30}-2 q^{34}-2 q^{36}+q^{40}+q^{42}\right)+t^{4}\left(q^{36}+q^{38}+q^{40}-q^{42}-q^{44}-q^{46}+q^{48}\right)\right)}{t^{12}\left(1-q^{2}\right)^{6}\left(1+2 q^{2}+q^{4}+q^{6}\right)^{2}}$

A.3. Specialization to $Q=-t q^{-2 N}$ : Some examples. In this section we consider the specialization $Q=-t q^{-2 N}$ for the case of the Hopf link colored by $\left(R_{1}, R_{2}\right)=$ $\left(1,1^{2}\right),\left(1^{2}, 1^{2}\right)$ and $\left(1^{3}, 1^{4}\right)$. We see that $G_{\lambda \mu}$ after this specialization is (up to an overall factor) a polynomial in $q$ and $t$ :

$$
\begin{aligned}
& G_{(1)\left(1^{2}\right)}(Q=-t, q, t)=G_{(1)\left(1^{2}\right)}\left(Q=-t q^{-2}, q, t\right)=0, \\
& G_{(1)\left(1^{2}\right)}\left(Q=-t q^{-4}, q, t\right)=-q^{-7} t^{-2}\left(1+q^{2}\right) \text {, } \\
& G_{(1)\left(1^{2}\right)}\left(Q=-t q^{-6}, q, t\right)=-q^{-13} t^{-2}\left(1+2 q^{2}+2 q^{4}+q^{6}+t^{2} q^{6}+t^{2} q^{8}+t^{2} q^{10}\right) \text {, } \\
& G_{(1)\left(1^{2}\right)}\left(Q=-t q^{-8}, q, t\right)=-q^{-19} t^{-2}\left(1+2 q^{2}+3 q^{4}+3 q^{6}+2 q^{8}+q^{10}\right. \\
& \left.+t^{2} q^{6}\left(1+2 q^{2}+3 q^{4}+3 q^{6}+2 q^{8}+q^{10}\right)\right) \text {, } \\
& G_{(1)\left(1^{2}\right)}\left(Q=-t q^{-10}, q, t\right)=-q^{-25} t^{-2}\left(1+2 q^{2}+3 q^{4}+\left(4+t^{2}\right) q^{6}\right. \\
& +\left(4+2 t^{2}\right) q^{8}+\left(3+4 t^{2}\right) q^{10}+\left(2+5 t^{2}\right) q^{12} \\
& \left.+\left(1+6 t^{2}\right) q^{14}+5 t^{2} q^{16}+4 t^{2} q^{18}+2 t^{2} q^{20}+t^{2} q^{22}\right), \\
& G_{(1)\left(1^{2}\right)}\left(Q=-t q^{-12}, q, t\right)=-q^{-32} t^{-2}\left(1+2 q^{2}+3 q^{4}+\left(4+t^{2}\right) q^{6}\right. \\
& +\left(5+2 t^{2}\right) q^{8}+\left(5+4 t^{2}\right) q^{10} \\
& +\left(4+6 t^{2}\right) q^{12}+\left(3+8 t^{2}\right) q^{14}+\left(2+9 t^{2}\right) q^{16} \\
& +\left(1+9 t^{2}\right) q^{18}+8 t^{2} q^{20}+6 t^{2} q^{22}+4 t^{2} q^{24} \\
& \left.+2 t^{2} q^{26}+t^{2} q^{28}\right) \text {. } \\
& G_{\left(1^{2}\right)\left(1^{2}\right)}\left(Q=-t q^{-2 N} q, t\right)=0, N=0,1, \\
& G_{\left(1^{2}\right)\left(1^{2}\right)}\left(Q=-t q^{-4}, q, t\right)=q^{-8} t^{-4}, \\
& G_{\left(1^{2}\right)\left(1^{2}\right)}\left(\left(Q=-t q^{-6}, q, t\right)=q^{-16} t^{-4}\left(1+q^{2}+\left(1+t^{2}\right) q^{4}+2 t^{2} q^{6}+2 t^{2} q^{8}+t^{2} q^{10}\right),\right. \\
& G_{\left(1^{2}\right)\left(1^{2}\right)}\left(Q=-t q^{-8}, q, t\right)=q^{-24} t^{-4}\left(1+q^{2}+\left(2+t^{2}\right) q^{4}+\left(1+3 t^{2}\right) q^{6}\right. \\
& +\left(1+5 t^{2}\right) q^{8}+6 t^{2} q^{10}+\left(5 t^{2}+t^{4}\right) q^{12} \\
& \left.+\left(3 t^{2}+t^{4}\right) q^{14}+\left(t^{2}+2 t^{4}\right) q^{16}+t^{4} q^{18}+t^{4} q^{20}\right)
\end{aligned}
$$




$$
\begin{aligned}
& G_{\left(1^{2}\right)\left(1^{2}\right)}\left(Q=-t q^{-10}, q, t\right)=q^{-32} t^{-4}\left(1+q^{2}+\left(2+t^{2}\right) q^{4}\right. \\
& +\left(2+3 t^{2}\right) q^{6}+\left(2+6 t^{2}\right) q^{8} \\
& +\left(1+9 t^{2}\right) q^{10}+\left(1+11 t^{2}+t^{4}\right) q^{12} \\
& +\left(11 t^{2}+2 t^{4}\right) q^{14} \\
& +\left(9 t^{2}+4 t^{4}\right) q^{16}+\left(6 t^{2}+5 t^{4}\right) q^{18}+\left(3 t^{2}+6 t^{4}\right) q^{20} \\
& \left.+\left(t^{2}+5 t^{4}\right) q^{22}+4 t^{4} q^{24}+2 t^{4} q^{26}+t^{4} q^{28}\right) \text {, } \\
& G_{\left(1^{2}\right)\left(1^{2}\right)}\left(Q=-t q^{-12}, q, t\right)=q^{-40} t^{-4}\left(1+q^{2}+\left(2+t^{2}\right) q^{4}+\left(2+3 t^{2}\right) q^{6}\right. \\
& +\left(3+6 t^{2}\right) q^{8}+\left(2+10 t^{2}\right) q^{10}+\left(2+14 t^{2}+t^{4}\right) q^{12} \\
& +\left(1+17 t^{2}+2 t^{4}\right) q^{14} \\
& +\left(1+18 t^{2}+5 t^{4}\right) q^{16} \\
& +t^{2}\left(17+7 t^{2}\right) q^{18}+t^{2}\left(14+11 t^{2}\right) q^{20} \\
& +2 t^{2}\left(5+6 t^{2}\right) q^{22} \\
& +2 t^{2}\left(3+7 t^{2}\right) q^{24}+3 t^{2}\left(1+4 t^{2}\right) q^{26} \\
& +\left(t^{2}+11 t^{4}\right) q^{28}+7 t^{4} q^{30} \\
& \left.+5 t^{4} q^{32}+2 t^{4} q^{34}+t^{4} q^{36}\right) \\
& G_{\left(1^{2}\right)\left(1^{2}\right)}\left(Q=-t q^{-14}, q, t\right)=q^{-48} t^{-4}\left(1+q^{2}+\left(2+t^{2}\right) q^{4}+\left(2+3 t^{2}\right) q^{6}\right. \\
& +\left(3+6 t^{2}\right) q^{8}+\left(3+10 t^{2}\right) q^{10} \\
& +\left(3+15 t^{2}+t^{4}\right) q^{12}+2\left(1+10 t^{2}+t^{4}\right) q^{14} \\
& +\left(2+24 t^{2}+5 t^{4}\right) q^{16} \\
& +\left(1+26 t^{2}+8 t^{4}\right) q^{18}+\left(1+13 t^{2}\left(2+t^{2}\right)\right) q^{20} \\
& +t^{2}\left(24+17 t^{2}\right) q^{22}+\left(20 t^{2}+22 t^{4}\right) q^{24} \\
& +3 t^{2}\left(5+8 t^{2}\right) q^{26} \\
& +2 t^{2}\left(5+13 t^{2}\right) q^{28}+6 t^{2}\left(1+4 t^{2}\right) q^{30} \\
& +t^{2}\left(3+22 t^{2}\right) q^{32}+\left(t^{2}+17 t^{4}\right) q^{34} \\
& \left.+13 t^{4} q^{36}+8 t^{4} q^{38}+5 t^{4} q^{40}+2 t^{4} q^{42}+t^{4} q^{44}\right),
\end{aligned}
$$




$$
\begin{aligned}
G_{\left(1^{3}\right)\left(1^{4}\right)}\left(Q=-t q^{-2 N}, q, t\right)= & 0, \quad N=0,1,2,3, \\
G_{\left(1^{3}\right)\left(1^{4}\right)}\left(Q=-t q^{-8}, q, t\right)= & -q^{-19} t^{-6}\left(1+q^{2}+q^{4}+q^{6}\right), \\
G_{\left(1^{3}\right)\left(1^{4}\right)}\left(Q=-t q^{-10}, q, t\right)= & -q^{-33} t^{-6}\left(1+2 q^{2}+3 q^{4}+\left(4+t^{2}\right) q^{6}\right. \\
& +\left(4+2 t^{2}\right) q^{8}+\left(3+4 t^{2}\right) q^{10} \\
& +\left(2+5 t^{2}\right) q^{12}+\left(1+6 t^{2}\right) q^{14}+5 t^{2} q^{16}+4 t^{2} q^{18} \\
& \left.+2 t^{2} q^{20}+t^{2} q^{22}\right),
\end{aligned}
$$

$G_{\left(1^{3}\right)\left(1^{4}\right)}\left(Q=-t q^{-12}, q, t\right)=-q^{-47} t^{-6}\left(1+2 q^{2}+4 q^{4}+\left(6+t^{2}\right) q^{6}\right.$

$$
\begin{aligned}
& +\left(8+3 t^{2}\right) q^{8}+\left(9+7 t^{2}\right) q^{10} \\
& +\left(9+12 t^{2}\right) q^{12}+\left(8+18 t^{2}\right) q^{14}+\left(6+23 t^{2}+t^{4}\right) q^{16}
\end{aligned}
$$$$
+\left(4+26 t^{2}+2 t^{4}\right) q^{18}+\left(2+26 t^{2}+4 t^{4}\right) q^{20}
$$$$
+\left(1+23 t^{2}+6 t^{4}\right) q^{22}
$$$$
+\left(18 t^{2}+8 t^{4}\right) q^{24}+\left(12 t^{2}+9 t^{4}\right) q^{26}
$$$$
+\left(7 t^{2}+9 t^{4}\right) q^{28}+\left(3 t^{2}+8 t^{4}\right) q^{30}
$$$$
\left.+\left(t^{2}+6 t^{4}\right) q^{32}+4 t^{4} q^{34}+2 t^{4} q^{36}+t^{4} q^{38}\right) \text {, }
$$

$G_{\left(1^{3}\right)\left(1^{4}\right)}\left(Q=-t q^{-14}, q, t\right)=-q^{-61} t^{-6}\left(1+2 q^{2}+4 q^{4}+\left(7+t^{2}\right) q^{6}\right.$

$$
\begin{aligned}
& +\left(10+3 t^{2}\right) q^{8}+\left(13+8 t^{2}\right) q^{10}+\left(16+15 t^{2}\right) q^{12} \\
& +\left(17+26 t^{2}\right) q^{14}+\left(17+38 t^{2}+t^{4}\right) q^{16}
\end{aligned}
$$$$
+\left(16+52 t^{2}+3 t^{4}\right) q^{18}+\left(13+7 t^{2}\left(9+t^{2}\right)\right) q^{20}
$$$$
+\left(10+72 t^{2}+13 t^{4}\right) q^{22}+\left(7+74 t^{2}+21 t^{4}\right) q^{24}
$$$$
+\left(4+72 t^{2}+30 t^{4}\right) q^{26}+\left(2+63 t^{2}+39 t^{4}\right) q^{28}
$$$$
+\left(1+52 t^{2}+46 t^{4}+t^{6}\right) q^{30}+t^{2}\left(38+50 t^{2}+t^{4}\right) q^{32}
$$$$
+2 t^{2}\left(13+25 t^{2}+t^{4}\right) q^{34}+t^{2}\left(15+t^{2}\right)\left(1+3 t^{2}\right) q^{36}
$$$$
+t^{2}\left(8+39 t^{2}+4 t^{4}\right) q^{38}+t^{2}\left(3+30 t^{2}+4 t^{4}\right) q^{40}
$$$$
+\left(t^{2}+21 t^{4}+5 t^{6}\right) q^{42}+t^{4}\left(13+4 t^{2}\right) q^{44}
$$$$
+t^{4}\left(7+4 t^{2}\right) q^{46}+3 t^{4}\left(1+t^{2}\right) q^{48}
$$$$
\left.+\left(t^{4}+2 t^{6}\right) q^{50}+t^{6} q^{52}+t^{6} q^{54}\right) \text {. }
$$ 


\section{References}

1. Khovanov, M.: A categorification of the Jones polynomial. Duke Math. J. 101, 359 (2000)

2. Ozsvath, P., Szabo, Z.: Holomorphic disks and knot invariants. Adv. Math. 186, 58 (2004)

3. Ozsvath, P., Szabo, Z.: "Holomorphic disks and link invariants." http://arxiv.org/abs/math/ 0512286v2[math.GT], 2007

4. Rasmussen, J.: "Floer homology and knot complements." Harvard thesis, http://arxiv.org/abs/math/ 0306378v1[math.GT], 2003

5. Khovanov, M.: sl(3) link homology. Algebr. Geom. Topol. 4, 1045 (2004)

6. Khovanov, M., Rozansky, L.: "Matrix factorizations and link homology." http://arxiv.org/abs/math/ 0401268v2[math.QA], 2004

7. Witten, E.: Quantum Field Theory And The Jones Polynomial. Commun. Math. Phys. 121, 351 (1989)

8. Gukov, S., Schwarz, A., Vafa, C.: Khovanov-Rozansky homology and topological strings. Lett. Math. Phys. 74, 53 (2005)

9. Dunfield, N., Gukov, S., Rasmussen, J.: The Superpolynomial for Knot Homologies. Experiment. Math. 15, 129 (2006)

10. Gukov, S., Walcher, J.: "Matrix Factorizations and Kauffman Homology." http://arxiv.org/abs/hep-th/ 0512298v1, 2005

11. Ooguri, H., Vafa, C.: Knot Invariants and Topological Strings. Nucl.Phys. B577, 419 (2000)

12. Witten, E.: Chern-Simons gauge theory as a string theory. Prog. Math. 133, 637 (1995)

13. Gopakumar, R., Vafa, C.: On the gauge theory/geometry correspondence. Adv. Theor. Math. Phys. 3, 1415 (1999)

14. Nekrasov, N.A.: Seiberg-Witten Prepotential From Instanton Counting. Adv. Theor. Math. Phys. 7, 831864 (2004)

15. Hollowood, T.J., Iqbal, A., Vafa, C.: Matrix Models, Geometric Engineering and Elliptic Genera. JHEP 0803, 069 (2008)

16. Khovanov, M., Rozansky, L.: "Matrix factorizations and link homology II." http://arxiv.org/abs/math/ 0505056v2[math.QA], 2006

17. Iqbal, A., Kozcaz, C., Vafa, C.: The Refined Topological Vertex. JHEP 0910, 069 (2009)

18. Aganagic, M., Klemm, A., Marino, M., Vafa, C.: The Topological Vertex. Commun. Math. Phys. 254, 425478 (2005)

19. Hori, K., Vafa, C.: "Mirror Symmetry.” http://arxiv.org/abs/math/hep-th/0002222v3, 2000

20. Aganagic, M., Klemm, A., Vafa, C.: Disk Instantons, Mirror Symmetry and the Duality Web. Z. Naturforsch. A 57, 1-28 (2002)

21. Ooguri, H., Vafa, C.: Worldsheet derivation of a large N duality. Nucl. Phys. B 641, 3 (2002)

22. Marino, M.: “Chern-Simons Theory, Matrix Models, And Topological Strings." Oxford: Oxford University Press, 2005

23. Labastida, J.M.F., Marino, M.: Polynomial invariants for torus knots and topological strings. Commun. Math. Phys. 217, 423 (2001)

24. Marino, M., Vafa, C.: "Framed knots at large N." http://arxiv.org/abs/hep-th/0108064v1, 2001

25. Taubes, C.: Lagrangians for the Gopakumar-Vafa conjecture. Geom. Topol. Monogr. 8, $73-95$ (2006)

26. Liu, K., Peng, P.: "Proof of the Labastida-Marino-Ooguri-Vafa Conjecture." http://arxiv.org/abs/0704. 1526v4[math.QA], 2009

27. Labastida, J.M.F., Marino, M., Vafa, C.: Knots, links and branes at large N. JHEP 0011, 007 (2000)

28. Khovanov, M., Rozansky, L.: "Virtual crossings, convolutions and a categorification of the $S O(2 N)$ Kauffman polynomial." http://arxiv.org/abs/math/0701333v1[math.QA], 2007

29. Iqbal, A.: "All genus topological string amplitudes and 5-brane webs as Feynman diagrams." http://arxiv. org/abs/hep-th/0207114v2, 2002

30. Okounkov, A., Reshetikhin, N., Vafa, C.: "Quantum Calabi-Yau and Classical Crystals". http://arxiv.org/ abs/hep-th/0309208v2, 2003

31. Gukov, S., Iqbal, A., Kozcaz, C., Vafa, C.: Work in progress

Communicated by N.A. Nekrasov 